\title{
Computational Conformal Geometry Behind Modern Technologies
}
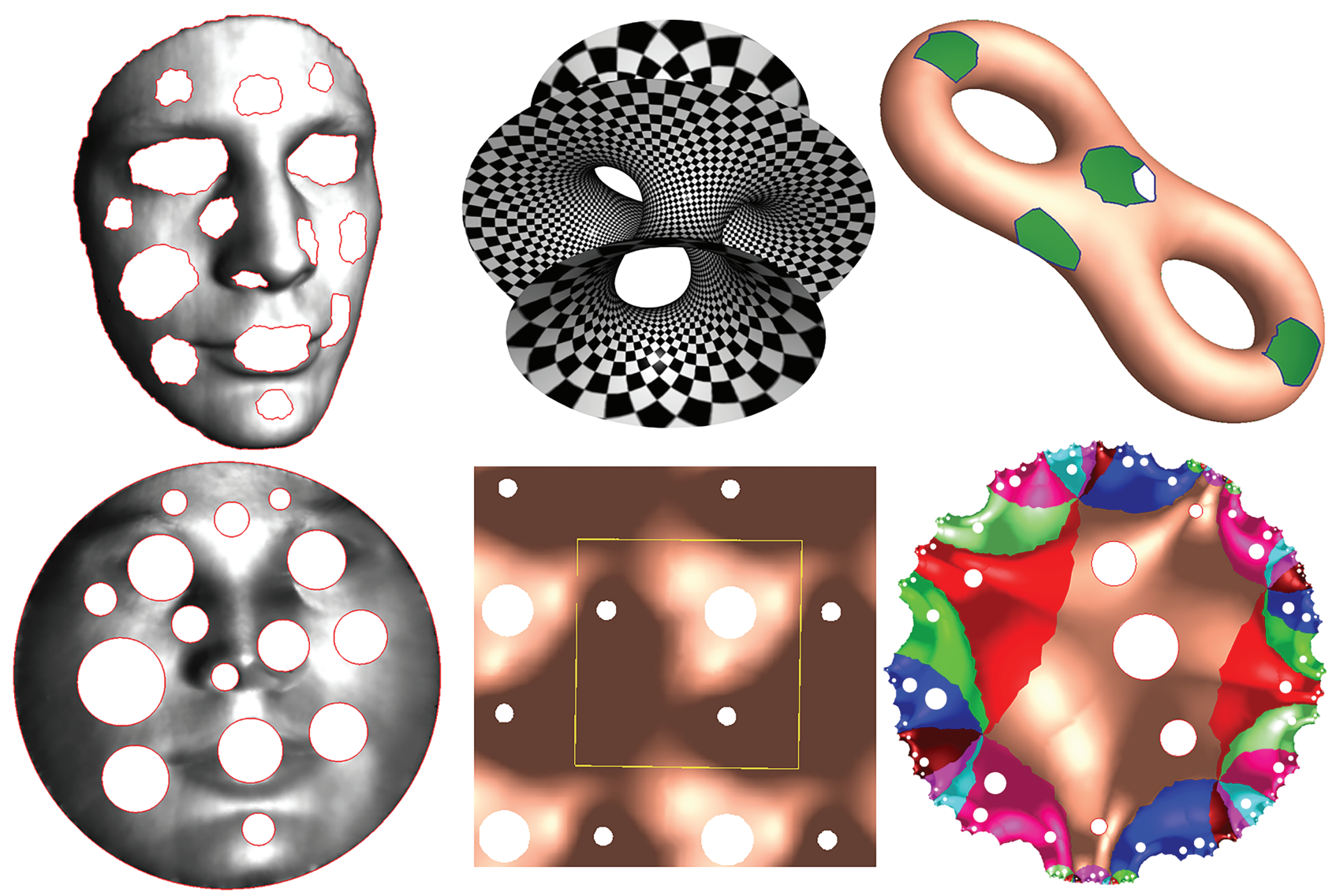

\section{Xianfeng Gu, Feng Luo, and Shing-Tung Yau}

Xianfeng David Gu is a SUNY Empire Innovation Associate Professor of Computer Science at Stony Brook University. His email address is gu@cs . stonybrook. edu.

Feng Luo is a Distinguished Professor of Mathematics at Rutgers University. His email address is fluo@math. rutgers. edu.

Shing-Tung Yau is a William Caspar Graustein Professor of Mathematics at Harvard University. His email address is yau@math . harvard. edu.

This work was supported in part by NSF 1737876, 1760527, 1811878, 1760471, 1762287, and 1737812.

Communicated by Notices Associate Editor Chikako Mese.

For permission to reprint this article, please contact:

reprint-permission@ams.org.

DOI: https://doi.org/10.1090/noti2164
Conformal geometry has deep roots in pure mathematics fields, such as Riemann surface theory, complex analysis, differential geometry, algebraic topology, partial differential equations and others. Historically, conformal geometry has been broadly used in many engineering applications [1], such as electro-magnetics, vibrating membranes, acoustics, elasticity, heat transfer and fluid flow. Most of these applications depend on conformal mappings between planar domains.

Recently, with the rapid development of 3D scanning and medical imaging technologies, 3D geometric data has become ubiquitous. Figure 2 shows a human facial surface acquired using a scanning system based on structured light. 

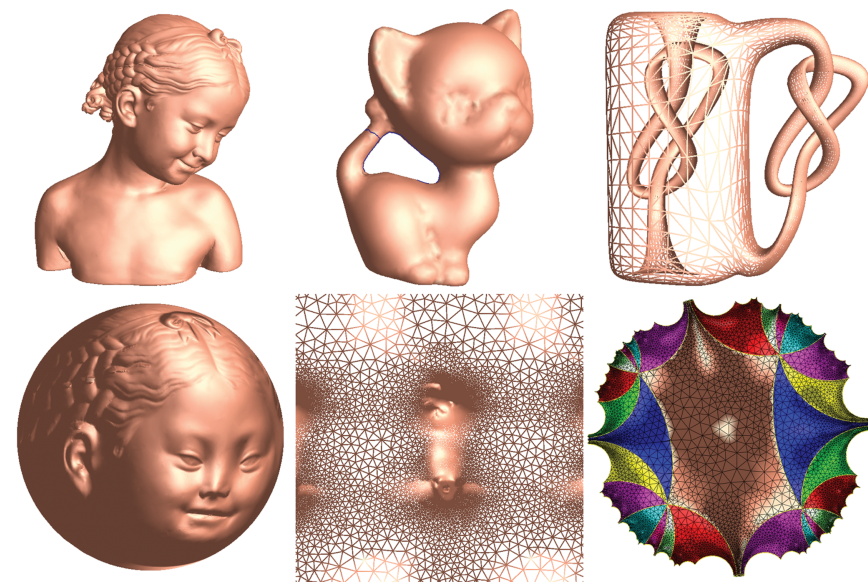

Figure 1. Surface uniformization, computed using harmonic mapping (left), Hodge decomposition (middle) and discrete surface Ricci flow (right) algorithms, respectively.

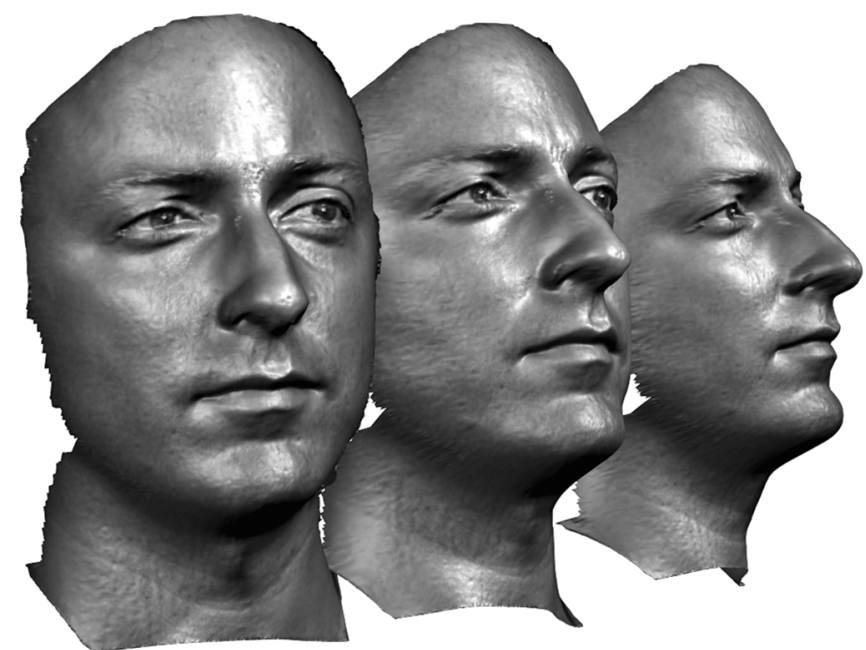

Figure 2. 3D human facial surface data scanned using structured light technology.

The system can capture dynamic geometric data with very high spacial resolution and scanning speed. It is challenging to process the huge amount of this geometric data with high accuracy and efficiency. The challenge can be tackled using various geometric theories. Compared to topology or Riemannian geometry, conformal geometry better fits this purpose because conformal structure has much richer information than topological structure and conformal mappings are much more flexible than isometries.

With the increase of computational power and further advances in mathematical theories, computational conformal geometry emerges as an interdisciplinary field, bridging mathematics and computer science. Computational conformal geometric theories and algorithms have been generalized from planar domains to surfaces with arbitrary topologies and have been applied to many engineering and medical fields. This paper is not intended to be an overview of the field and will mainly focus on our contributions to the field. Many important works have not been touched upon and many references are missing due to the page limit imposed by the Notices. More details can be found, for instance, in [12]. A more complete list of references can be found at the authors' web pages.

\section{Fundamental Concepts}

Riemann surfaces. The underlying spaces for 2-dimensional conformal geometry are Riemann surfaces. Roughly speaking, a Riemann surface is a topological surface on which the notation of angle can be defined. More precisely, given a surface $S$, a complex structure on $S$ is a special collection of coordinate charts $\left\{\left(U_{i}, \varphi_{i}\right) \mid i \in I\right\}$ such that $S=\bigcup_{i} U_{i}$ and the transition functions $\varphi_{i} \circ \varphi_{j}^{-1}$ are biholomorphic maps for all choices of indices $i, j$. (Similarly, if all transition functions $\varphi_{i} \circ \varphi_{j}^{-1}$ are smooth, then the collection of coordinate charts is called a smooth structure.) A Riemann surface is a topological surface together with a complex structure. Since biholomorphic maps are orientation preserving and angle preserving, each Riemann surface is oriented and one can naturally measure the angle between two intersecting curves on a Riemann surface. Furthermore, since the composition of a harmonic function and a holomorphic function is again harmonic, the notions of harmonic functions and, more generally, harmonic and holomorphic differentials are well defined on a Riemann surface.

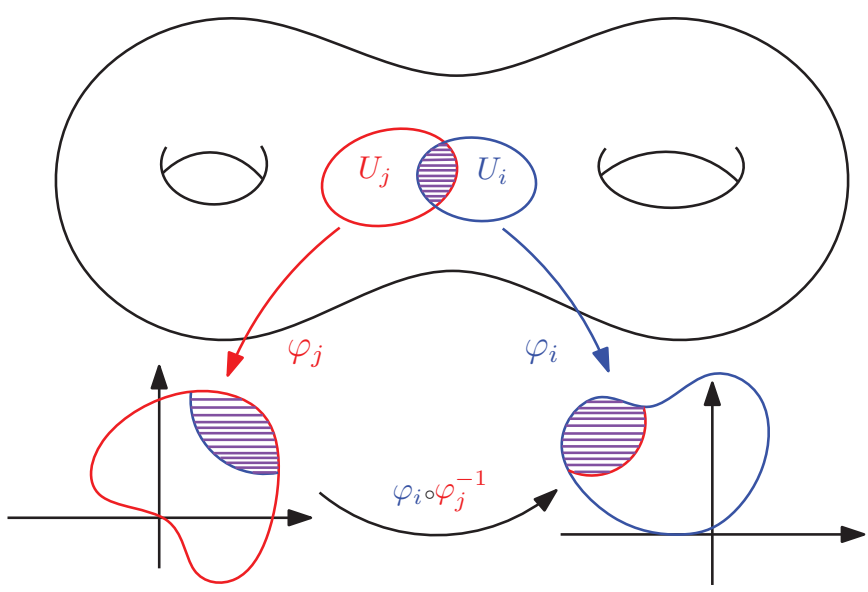

Figure 3. Coordinate charts.

Almost every surface we encounter is a Riemann surface. For instance, every open set in the plane is a Riemann surface. In fact, complex analysis that we learn in undergraduate and graduate courses is the Riemann surface theory on open sets in the plane $\mathbb{C}$. Futhermore, every oriented smooth surface $S$ with a Riemannian metric $\mathbf{g}$ is naturally a Riemann surface-the complex structure on $S$ is induced by the Riemannian metric $\mathbf{g}$ and the notion of angle 

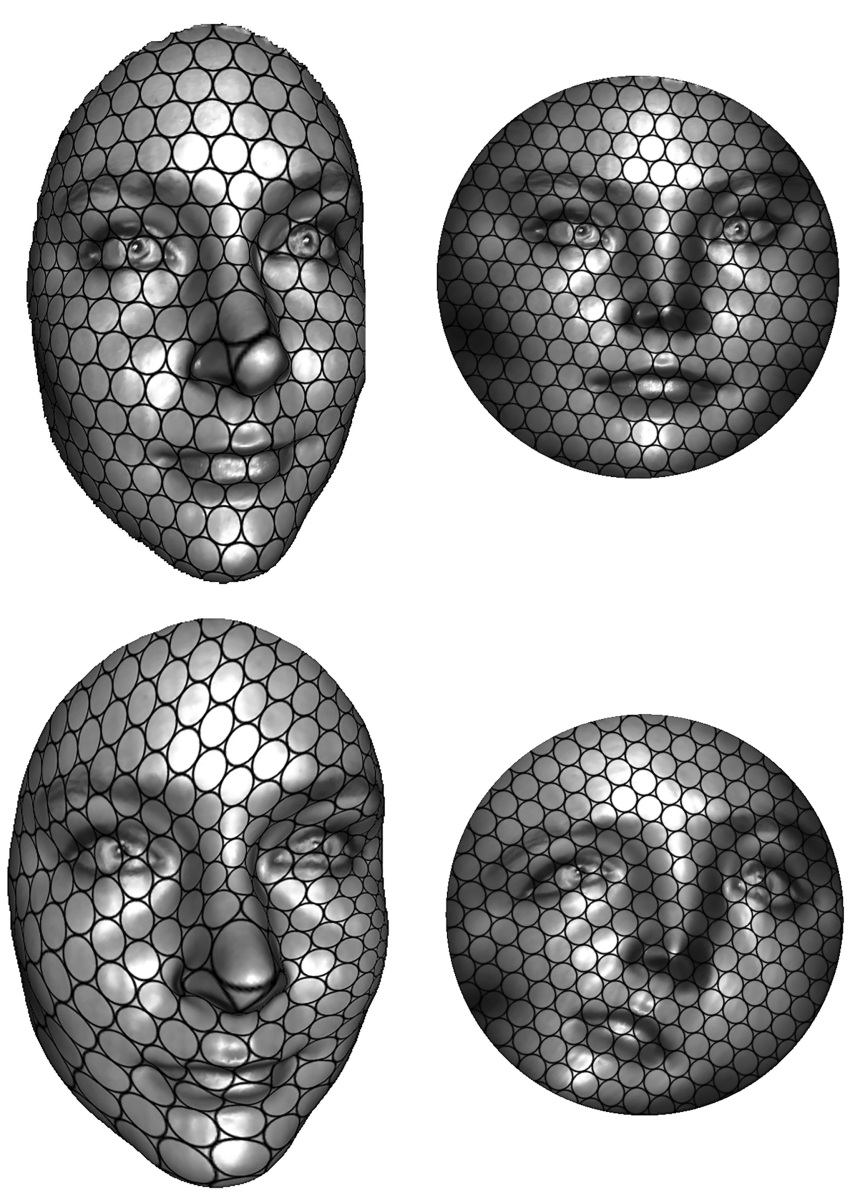

Figure 4. Top row: conformal mapping transforms infinitesimal circles to infinitesimal circles; bottom row: general diffeomorphism maps infinitesimal ellipses to infinitesimal circles.

defined by $\mathbf{g}$ and by the complex structure coincide. This was first observed by C. F. Gauss for the case of real analytic Riemannian metrics. He showed that at each point $p \in S$, one can find a coordinate chart $(U, \phi)$ such that $\phi:(U, \mathbf{g}) \rightarrow\left(\mathbb{R}^{2}, d x^{2}+d y^{2}\right)$ is an angle-preserving smooth embedding. These coordinate charts $(U, \phi)$ are called the isothermal coordinates. In particular, all smooth orientable surfaces in 3-space are naturally Riemann surfaces. Another class of Riemann surfaces comes from algebraic geometry. Namely, an algebraic curve in $\mathbb{C}^{2}$, i.e., a surface defined by a polynomial equation $p(z, w)=0$, is naturally a Riemann surface where coordinate charts are derived from the implicit function theorem.

Conformal maps. The natural correspondences between Riemann surfaces are those bijections that preserve angles. We call them conformal maps. From complex analysis, we know that holomorphic maps are angle preserving (away from singularities). Thus, conformal maps can be considered as generalizations of injective holomorphic maps.
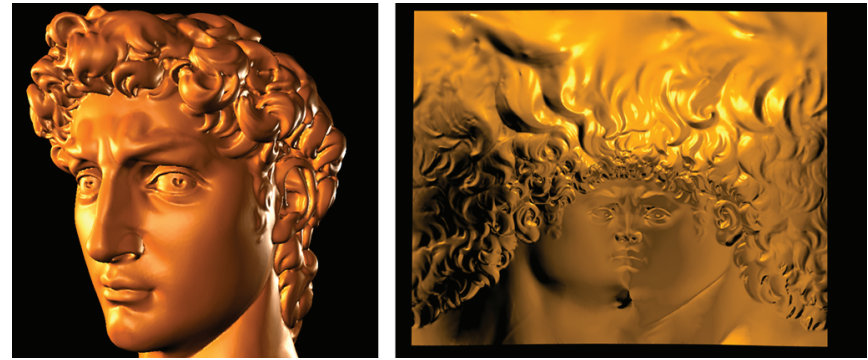

Figure 5. Conformal mapping preserves local shapes.

A prominent example of a conformal map is the stereographic map from the unit sphere to the plane.

Conformal maps can also be characterized as those smooth maps which preserve infinitesimal circles. In Figure 4, two diffeomorphisms map a female facial surface to the planar unit disk. The top row shows a conformal mapping, which infinitesimally maps circles on the face to the infinitesimal circles on the disk. In contrast, the bottom illustrates a general diffeomorphism which maps infinitesimally ellipses to circles and not vice versa. If the eccentricities of the ellipses (the ratio between the major axis and the minor axis) are uniformly bounded, then the mapping is called a quasi-conformal map.

Equivalently, a conformal map preserves local shapes, namely locally it is a scaling transformation followed by a rotation, where the scaling factor varies from point to point. This is illustrated in Figure 5. The head surface of Michelangelo's David sculpture is conformally flattened onto a planar rectangle. The complicated curved surface becomes a planar sheet under this conformal map. From the shading, one can see that the complicated local geometric shapes, such as the eyes, ears and curly hair, are well recognizable on the plane.
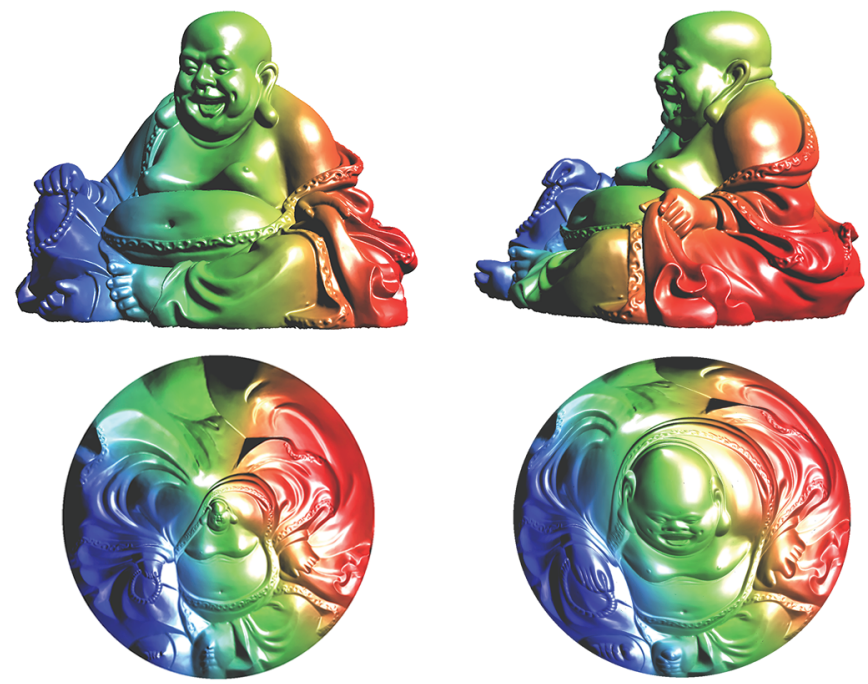

Figure 6. Comparison between an angle-preserving mapping (left) and an area-preserving mapping (right) from a Buddha surface onto a planar disk. 
In engineering applications, the distortions of mappings are classified into two categories, angle distortion and area distortion. It is always desirable to find the optimal mappings that minimize distortions. A conformal mapping preserves angles, but distorts areas. The area distortion is called the conformal factor induced by the mapping. Depending on the topology and the geometry of the surface, the distortion of area by a conformal map could be drastic. Figure 6 compares a conformal mapping (left) and an area-preserving mapping (right) from a Buddha surface to the planar unit disk. It can be seen that the conformal mapping induces large area deformations in the head region, whereas the area-preserving mapping induces large angle deformations along the boundary of the Buddha surface. If a mapping preserves both angle and area, then it is isometric and preserves the Gaussian curvature. Hence, there does not exist a mapping from the Buddha surface to the planar disk that preserves both angle and area.

Uniformization. The famous Riemann mapping theorem classifies simply connected planar domains up to conformal diffeomorphism. Can one classify all Riemann surfaces up to conformal diffeomorphisms? This classification is achieved by the remarkable uniformization theorem of Poincare and Koebe proved in 1907. It states that every simply connected Riemann surface is conformally diffeomorphic to the 2-sphere $\mathbb{S}^{2}$, the plane $\mathbb{E}^{2}$ or the open unit disc $\mathbb{H}^{2}$, as shown in Figure 1. Using covering space theory, the uniformization theorem implies that every connected oriented surface with a Riemannian metric $(S, \mathbf{g})$ is conformally diffeomorphic to one of three canonical models of surfaces: (i) the unit sphere $\mathbb{S}^{2}$, (ii) a flat torus, or $\mathbb{E}^{2}$, or $\mathbb{E}^{2}-\{0\}$ or (iii) a hyperbolic surface $\mathbb{U}^{2} / \Gamma$, where $\Gamma$ is a discrete torsion-free subgroup of isometries of the hyperbolic plane $\mathbb{H}^{2}$. Equivalently, the uniformization theorem states that for any connected Riemannian surface $(S, \mathbf{g})$ there exists a real-valued function $\lambda: S \rightarrow \mathbb{R}$ such that the conformal Riemannian metric $e^{2 \lambda} \mathbf{g}$ is a complete Riemannian metric of constant Gaussian curvature 1, 0 or -1 . The three curvatures correspond to the three cases (i), (ii) and (iii) above. The uniformization theorem also holds for compact surfaces with boundaries. As shown in Figure 7, Riemannian metric surfaces with boundaries can be conformally mapped to the canonical surfaces with constant curvatures with a finite number of geodesic disks removed. We remark that there is still a famous open problem on conformal classification of planar domains. In 1910, P. Koebe conjectured that every connected open set in the plane is conformally diffeomorphic to a new domain whose boundary components are either round circles or points.

The uniformization theorem plays a fundamental role for applications in engineering and medical imaging. It sorts all kinds of shapes in the real physical world to only
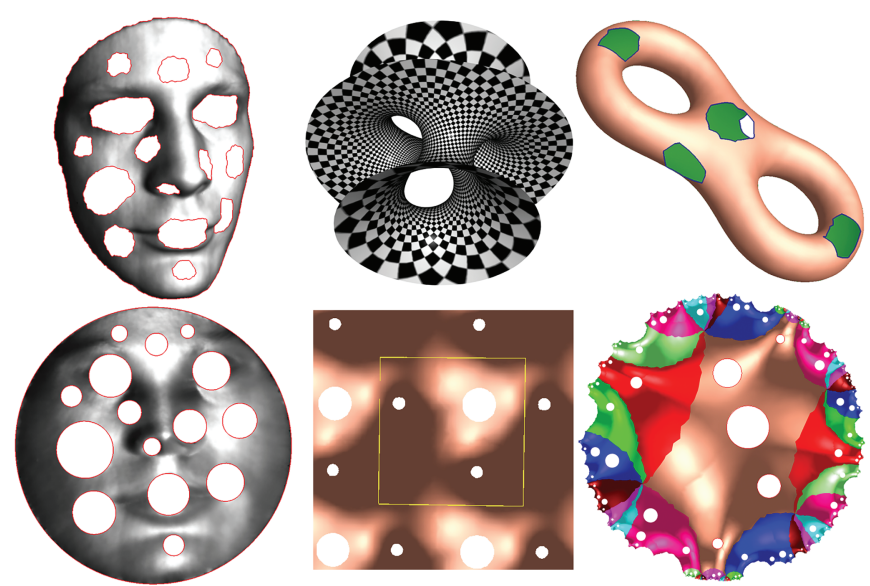

Figure 7. Uniformization for surfaces with boundaries.

three canonical types. If one can develop an algorithm that can handle the canonical type surfaces, then the algorithm can process all shapes via uniformization. This greatly simplifies the algorithmic design task for engineers.

\section{Computational Methods}

With the advances of modern technologies, surfaces are produced digitally at an alarming rate these days. There is an urgent need to process and categorize them. A useful form of these digital surfaces is polyhedral surfaces. In Figure 8 Michelangelo's David sculpture surface is approximated by a polyhedral surface. Classical differential geometric theories are inadequate to deal with polyhedral and digital surfaces. It is a major challenge to develop computable theories for conformal, harmonic, quasi-conformal, isometric, area-preserving and other mappings for polyhedral surfaces. We will present our approaches to this challenge below. There are several guiding principles one tries to follow in discretizing classical concepts. Firstly, the discrete counterparts should have their own intrinsic geometric structures. Secondly, there should be a finite-dimensional variational principle whose critical points would correspond to the discrete entities. As usual, a finite-dimensional variational characterization will then lead to practical computational
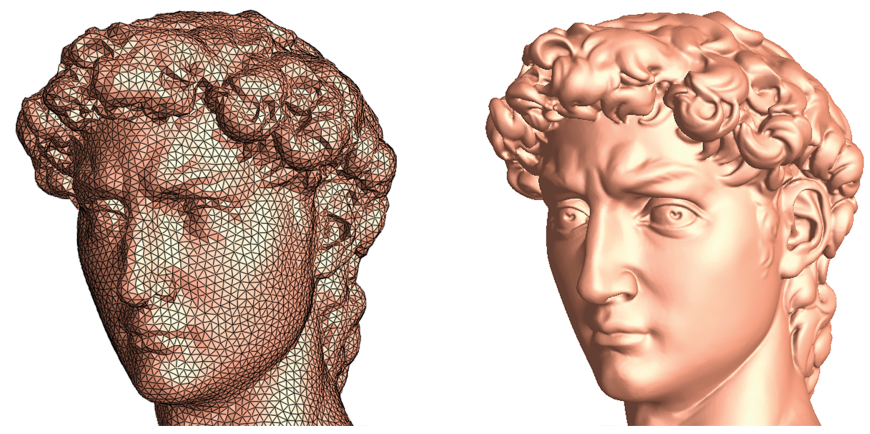

Figure 8. Discrete representation of Michelangelo's David sculpture surface. 
algorithms with efficiency, accuracy and robustness. Finally, the discrete entities should converge to their smooth counterparts when the triangular meshes are suitably chosen.

There are three categories of computational algorithms, as illustrated in the computation of surface uniformization Figure 1: the harmonic maps method in the left frame, the Hodge decomposition and meromorphic differential method in the middle frame and the discrete surface Ricci/Yamabe flow method in the right frame. Different methods have different advantages and disadvantages, and are able to solve different problems. None of them can be replaced by others. For example, in order to find the conformal hyperbolic metric, discrete curvature flow should be used; in order to compute holomorphic differentials, the Hodge decomposition method should be applied and so on.

Concepts in discrete setting. Let us begin by recalling what triangulations and polyhedral surfaces are. Take a collection of Euclidean triangles and identify pairs of edges by homeomorphisms. The quotient space is a topological surface $\Sigma$ together with a triangulation $\mathcal{T}$. Triangles in $\mathcal{T}$ come from the quotients of the Euclidean triangles. If we identify pairs of edges by isometries (i.e., length-preserving homeomorphisms), we obtain a polyhedral metric, or piecewise linear (PL) metric, on the triangulated surface $(\Sigma, \mathcal{T})$. A polyhedral surface is a surface with a PL metric. For instance, the boundary of a 3-dimensional polytope in the 3-space carries a natural PL metric. Clearly a PL metric $d$ on $(\Sigma, \mathcal{T})$ can be determined by the edge length function $\ell: E(\mathcal{T}) \rightarrow \mathbb{R}_{>0}$ which records the length of an edge $e$ in the set $E(\mathcal{T})$ of all edges in $\mathcal{T}$. The function $\ell$ must only satisfy the triangle inequality, that is, if $e_{i}, e_{j}, e_{k}$ form the edges of a triangle, then $\ell\left(e_{i}\right)+\ell\left(e_{j}\right)>\ell\left(e_{k}\right)$. Therefore, a PL metric can be coded by a computer easily. We consider a PL metric $d: \Sigma \times \Sigma \rightarrow \mathbb{R}_{\geq 0}$ as a metric in the sense of point set topology. The distance $d(x, y)$ between two points $x, y \in \Sigma$ is the infimum of the lengths of all paths on $\Sigma$ joining $x$ and $y$. Here the length of a path inside each triangle is computed using the Euclidean metric induced by the edge lengths of the triangle. It follows that the PL metric $d$ is flat away from the vertices of $\mathcal{T}$. Let $V(\mathcal{T})$ be the set of all vertices in the triangulation $\mathcal{T}$. At each vertex $v \in V(\mathcal{T})$, the discrete curvature $K_{d}(v)$ of $d$ is $2 \pi$ less the sum of all angles at vertex $v$. See Figure 10 . There may be many different triangulations $\mathcal{T}^{\prime}$ and the associated length functions $l^{\prime}: E\left(\mathcal{T}^{\prime}\right) \rightarrow \mathbb{R}_{>0}$ describing the same PL metric $d$. One of the goals is to develop a computable discrete counterpart of conformal geometry which is independent of the choice of triangulations.

There are now several ways to define the discrete conformal equivalence of PL metrics on a surface. In this paper, we will focus on two such definitions. A more general form

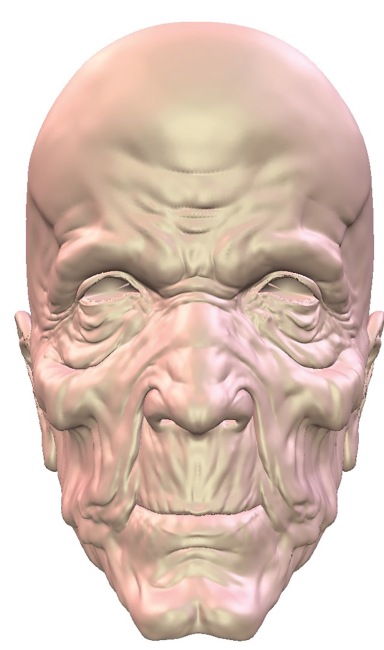

(a) original surface

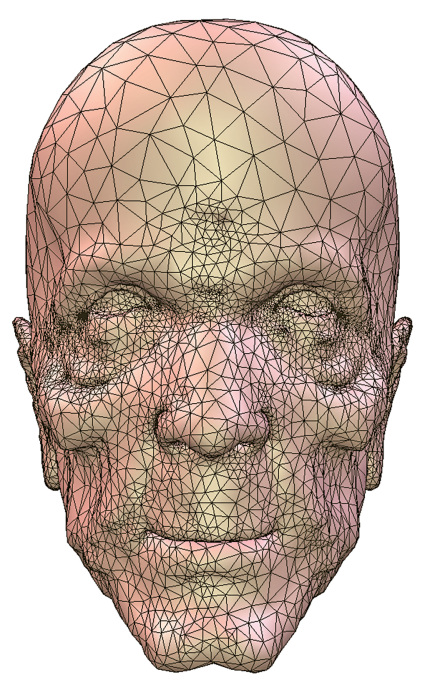

(c) $2 \mathrm{k}$ samples

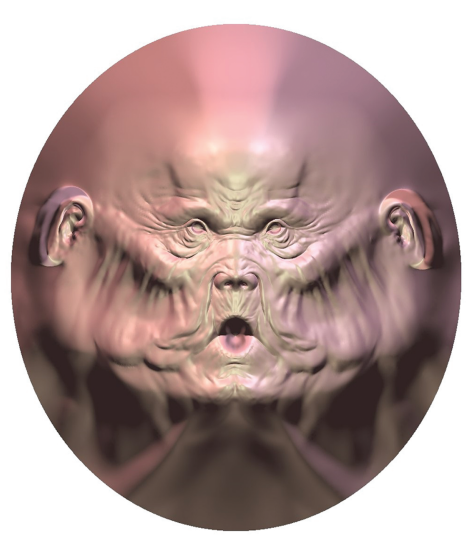

(b) conformal mapping

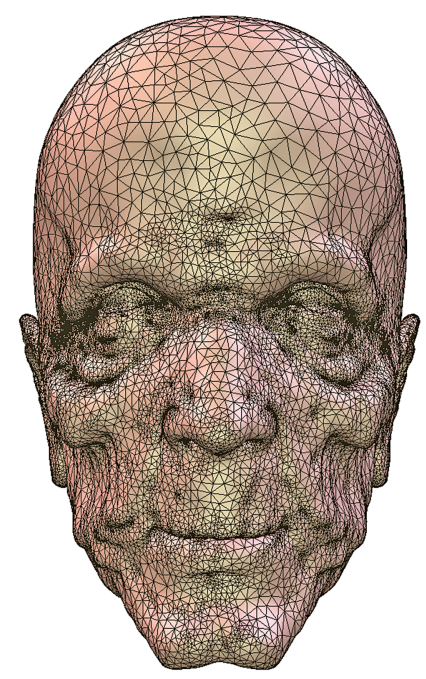

(d) $8 \mathrm{k}$ samples
Figure 9. Geometric approximation using Riemann mapping and normal cycle.

of discrete conformal equivalences, which includes these two as special cases, was proposed by D. Glickenstein [6] Both of these definitions were motivated by the seminal work of R. Hamilton on Ricci flows. On a Riemannian surface, the Ricci flow is defined as $\frac{\partial \mathbf{g}(t)}{\partial t}=-2 K_{\mathbf{g}(t)} \mathbf{g}(t)$, where $K_{g}$ is the Gaussian curvature. The flow preserves the conformal class of a Riemannian surface.

\begin{tabular}{|l|r|}
\hline Smooth category & Discrete category \\
\hline \hline smooth surfaces $S$ & triangulated surf. $(\Sigma, \mathcal{T})$ \\
\hline functions on $S$ & functions on $V(\mathcal{T})$ \\
\hline Riemannian metric $\mathbf{g}$ & PL metric $\mathbf{d}$ on $(\Sigma, \mathcal{T})$ \\
\hline Gaussian curvature of $\mathbf{g}$ & disc. curv. $K_{d}$ on $V(\mathcal{T})$ \\
\hline conformal class $\left\{e^{u} \mathbf{g}\right\}$ & disc. conformal class of $\mathbf{d}$ \\
\hline
\end{tabular}

Table 1. Corresponding concepts on smooth surfaces and polyhedral surfaces. 


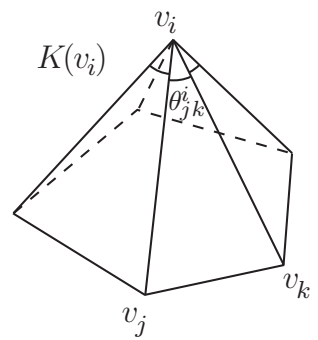

(a) discrete curvature $K\left(v_{i}\right)=2 \pi-\sum_{j k} \theta_{j k}^{i}$

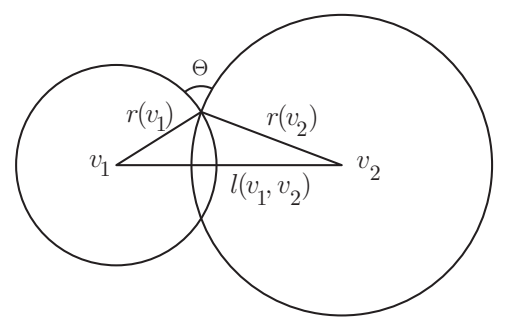

(b) circle packing

Figure 10. Discrete curvature and circle packing.

Table 1 summarizes common smooth notions and their discrete counterparts.

Discrete conformal geometry and discrete Yamabe flow. Given two PL metrics on a triangulated surface $(\Sigma, \mathcal{T})$ whose edge length functions are $\ell$ and $\hat{\ell}$, we say $\ell$ and $\hat{\ell}$ are related by a vertex scaling [15] and [18], written as $\hat{\ell}=u * \ell$, if there exists a function $u: V(\mathcal{T}) \rightarrow \mathbb{R}$ such that for each edge $e$ with endpoints $v_{1}, v_{2}$,

$$
\hat{\ell}(e)=e^{u\left(v_{1}\right)+u\left(v_{2}\right)} \ell(e) .
$$

Equation (1) represents a discretization of the conformal Riemannian metric $e^{2 u} \mathbf{g}$. It is proved in [9] that if $\mathbf{g}$ is a Riemannian metric on a compact manifold $M$ and $u$ : $M \rightarrow \mathbb{R}$ is a smooth function, then there exists a constant $C>0$ such that for any pairs of points $x, y \in M$, $\left|d_{e^{4 u} \mathbf{g}}(x, y)-e^{u(x)+u(y)} d_{\mathbf{g}}(x, y)\right| \leq C d_{\mathbf{g}}(x, y)^{3}$. Here $d_{\mathbf{g}}$ is the Riemannian distance associated to the Riemannian metric $\mathbf{g}$, i.e., $d_{\mathbf{g}}(x, y)$ is the infimum of the lengths of all paths joining $x$ to $y$. The above estimate holds the key for showing that discrete conformal maps defined using (1) converge to the smooth case.

The definition (1) carries a natural variational principle relating a PL metric to its discrete curvature [15]. Fix a Euclidean triangle whose $i$ th edge joins the $j$ th and $k$ th vertices and has length $l_{i},\{i, j, k\}=\{1,2,3\}$. Let $\Delta$ be the new triangle whose $i$ th edge length is $e^{u_{j}+u_{k}} l_{i}$, and let $a_{i}$ be the angle of $\Delta$ at the $i$ th vertex. Then the Jacobian matrix $\left[\frac{\partial a_{i}}{\partial u_{j}}\right]_{3 \times 3}$ is symmetric and negative semidefinite. In particular, the locally concave function $F\left(u_{1}, u_{2}, u_{3}\right)=$ $\int_{0}^{u} \sum_{i=1}^{3} a_{i} d u_{i}$ is well defined and satisfies $\frac{\partial F}{\partial u_{i}}=a_{i}$. Note that discrete curvature is built from the inner angles $a_{i}$ 's. The above formula thus relates a PL metric $u * \ell$ and its discrete curvature. The explicit form of the function $F$ was found in the work of Bobenko-Pinkall-Springborn [1]. They showed that $F$ can be extended to a concave function on $\mathbb{R}^{3}$ and is related to the 3-dimensional hyperbolic volume of ideal tetrahedra.

A basic goal in geometry is to find the relationship between the metric and its curvature. In the discrete setting, it translates into the following questions. Given a

polyhedral metric with edge-length function $\ell$ on a closed triangulated surface $(\Sigma, \mathcal{T})$ and a function $\hat{K}: V(\mathcal{T}) \rightarrow$ $(-\infty, 2 \pi)$, can one find $u: V(\mathcal{T}) \rightarrow \mathbb{R}$ such that $u * \ell$ is still an edge-length function on $\mathcal{T}$ and its curvature $K_{u * \ell}$ is the given function $\hat{K}$ ? Obviously the function $\hat{K}$ must satisfy the Gauss-Bonnet condition $\sum_{v \in V(\mathcal{T})} \hat{K}(v)=2 \pi \chi(\Sigma)$. If such a function $u$ exists, then any other function that differs from $u$ by a constant is also a solution of the problem. The next question is: is the function $u$ unique up to the addition of a constant? Suppose one can solve the prescribing curvature equation $K_{u * \ell}=\hat{K}$, how to find $u$ effectively?

These questions, together with Hamilton's Ricci flow, led to the introduction of the discrete Yamabe flow [15]:

$$
\frac{d u(t)}{d t}(v)=\hat{K}(v)-K_{u * \ell}(v) .
$$

The variational principle associated to (1) shows that the flow is the gradient flow of the locally concave discrete energy

$$
E(u)=\int_{0}^{u} \sum_{v \in V(\mathcal{T})}\left(\hat{K}(v)-K_{u * \ell}(v)\right) d u(v) .
$$

It is proved in [1] that the solution to the equation $K_{u * \ell}=$ $\hat{K}$ is unique in $u$ up to the addition of a constant function. However, the existence of $u$, even if one assumes the GaussBonnet condition on $\hat{K}$, is false and the discrete Yamabe flow develops singularities in finite time.

The drawback of (1) is that it depends on the choices of the triangulation $\mathcal{T}$. Recall that a marked surface is a pair $(S, V)$, where $V$ is a finite set in $S$. A PL metric on $(S, V)$ is a PL metric on $S$ such that its conical singularities are contained in $V$. By a triangulation $\mathcal{T}$ of $(S, V)$, we mean a triangulation of $S$ such that $V(\mathcal{T})=V$. Suppose $d_{1}$ and $d_{2}$ are two PL metrics on a marked surface $(\Sigma, V)$ and $\mathcal{T}$ and $\mathcal{T}^{\prime}$ are two triangulations of $(\Sigma, V)$. Let $\ell_{i}$ and $\ell_{i}^{\prime}$ be the associated edge length functions of $d_{i}$ for $\mathcal{T}$ and $\mathcal{T}^{\prime}$. If $\ell_{1}=u * \ell_{2}$, does it follow that $\ell_{1}^{\prime}=w * \ell_{2}^{\prime}$ for some $w \in \mathbb{R}^{V}$ ? An affirmative answer would imply that (1) is independent of the choice of triangulations. Unfortunately, the answer is negative. However, the condition $\ell_{1}^{\prime}=w * \ell_{2}^{\prime}$ does hold for some $w$ if we assume that all triangulations $\mathcal{T}$ and $\mathcal{T}^{\prime}$ are Delaunay in $d_{i}$ [8]. Recall that a Delaunay triangulation of a polyhedral surface is a geometric triangulation such that for each edge the sum of two angles facing the edge is at most $\pi$. Given a PL metric $d$ on a marked surface $(S, V)$, there is always a Delaunay triangulation of $(\Sigma, V, d)$ whose vertex set is $V$. Generically, Delaunay triangulation on $(\Sigma, V, d)$ is unique. However, nonuniqueness occurs when the sum of the two angles facing an edge $e$ equals $\pi$. In this case, consider the quadrilateral $Q$ formed by the two triangles adjacent to $e$ and replace the diagonal $e$ in $Q$ by the other diagonal. The resulting triangulation is still Delaunay and the operation is called an edge flip. Note 
that edge flip does not change the underlying PL metric, but only the combinatorics.

A triangulation-independent definition of discrete conformal equivalence of PL metrics on a marked surface $(\Sigma, V)$ was introduced in [8] by modifying (1) and adding the Delaunay condition on triangulations. Two PL metrics $d_{1}$ and $d_{2}$ on $(\Sigma, V)$ are said to be related by a move if one can find Delaunay triangulations $\mathcal{T}_{i}$ of $d_{i}$ such that either $\mathcal{T}_{1}=\mathcal{T}_{2}$ and their associated edge-length functions $l_{d_{1}}$ and $l_{d_{2}}$ differ by (1) on $\mathcal{T}_{1}$, or $d_{1}=d_{2}$ and $\mathcal{T}_{1}$ differs from $\mathcal{T}_{2}$ by an edge flip. Two PL metrics on $(\Sigma, V)$ are discrete conformal if they are related by a finite sequence of moves. It turns out this is the correct notion to solve both the existence and uniqueness questions.

Theorem 1 ([8]). Suppose $d$ is a PL metric on a compact connected surface $(\Sigma, V)$ and $\hat{K}: V \rightarrow(-\infty, 2 \pi)$ is any function such that $\sum_{v \in V} \hat{K}(v)=2 \pi \chi(\Sigma)$. Then there exists a PL metric $d^{*}$, unique up to scaling, on $(\Sigma, V)$ such that $d^{*}$ is discrete conformal to $d$ and its discrete curvature satisfies $K_{d^{*}}=\hat{K}$. Furthermore, $d^{*}$ can be found using a finite-dimensional convex variational principle.

Take the function $\hat{K}$ to be the constant $\frac{2 \pi \chi(\Sigma)}{|V|}$. Then the theorem implies each PL metric $d$ is discrete conformal to a unique (up to scaling) PL metric $d^{*}$ of constant discrete curvature. This can be considered as a discrete version of the uniformization theorem. The above theorem for the case of the torus was first proved by F. Fillastre [5] in a different context.

There is a hyperbolic geometric interpretation of the above discrete conformal equivalence which makes the geometric picture clear. For more details, see [8], [7] and [1].

The convergence of discrete conformal metrics to the Poincaré metric on the torus was established in [9] and on high genus surfaces by T. Wu and X. Zhu recently.

Discrete conformal geometry and discrete Ricci flow. The edge flip operation used in the above discrete conformal equivalence relation has created computational complications. There is a more robust, triangulation dependent discrete curvature flow which one can use to find PL metrics with the targeted curvatures. The basic idea comes from W. Thurston's work on circle packing and Hamilton's work on Ricci flow. See [19] for details on circle packing. This new discretization focuses on the circle-preserving property of the conformal maps. The associated finitedimensional variational principle was first established by Colin de Verdiere [4] in the tangential case and in the general case in [3]. Based on the variational principle, the work [3] introduced discrete Ricci flow (see (3)) on surfaces and established its basic properties. Algorithmic details can be found in [16] and [22].
Here are some mathematical details. Given a triangulated surface $(S, \mathcal{T})$ and an assignment of edge weight $\Theta: E(\mathcal{T}) \rightarrow[0, \pi)$ (measuring the intersection angles of circles), a circle packing metric is a function, called radius assignment, $r: V(\mathcal{T}) \rightarrow \mathbb{R}_{>0}$ such that the associated length function

$$
l\left(v_{1} v_{2}\right)=\sqrt{r\left(v_{1}\right)^{2}+r\left(v_{2}\right)^{2}+2 r\left(v_{1}\right) r\left(v_{2}\right) \cos \left(\Theta\left(v_{1} v_{2}\right)\right)}
$$

produces a PL metric on $(S, \mathcal{T})$, i.e., satisfies the triangular inequality $l\left(e_{i}\right)+l\left(e_{j}\right)>l\left(e_{k}\right)$ for every trip of edges $\left\{e_{i}, e_{j}, e_{k}\right\}$ belonging to a triangle in $\mathcal{T}$. Thurston proved that if $\Theta(E(\mathcal{T})) \subset[0, \pi / 2]$ (see Figure $10(\mathrm{~b})$ ), then the triangle inequality always holds for all choices of $r \in \mathbb{R}_{>0}^{V(\mathcal{T})}$ (see [20]). The discrete curvature $K_{r}$ of $r$ is defined to be the discrete curvature of the PL metric induced by $l$. In this setting, a discrete conformal class is defined as the set of all PL metrics induced by different choices of $r: V(\mathcal{T}) \rightarrow \mathbb{R}_{>0}$ for a fixed edge weight $\Theta$. Since different choices of $r$ amount to different sizes of circles at vertices, this discretization captures the circle-preserving properties of the conformal maps.

The basic questions are for a fixed prescribed $\Theta$ to find a radius assignment $r \in \mathbb{R}^{V(\mathcal{T})}$ such that its curvature $K_{r}$ is a prescribed function $\hat{K}$ and to determine if $r$ is unique up to scaling. For $\Theta(E(\mathcal{T})) \subset[0, \pi / 2]$, both of them were solved by W. Thurston in his famous notes [20]. He proved that $r$ is unique up to scaling and found the necessary and sufficient conditions on $\hat{K}$ to be solvable by $r$. Most remarkably the conditions discovered by Thurston on $\hat{K}$ are the Gauss-Bonnet (linear) equation and a finite set of linear inequalities.

The variational principle associated to the circle packing takes the following form. Fix $\Phi_{1}, \Phi_{2}, \Phi_{3} \in[0, \pi)$. Suppose a triangle $\Delta$ has edge lengths $l_{1}, l_{2}, l_{3}$ of the form given by $l_{i}^{2}=r_{j}^{2}+r_{k}^{2}+2 r_{j} r_{k} \cos \left(\Theta_{i}\right)$ and $r_{i}=e^{u_{i}}$. If one denotes the angles of $\Delta$ by $a_{i}$, then the Jacobian matrix $\left[\frac{\partial a_{i}}{\partial u_{j}}\right]_{3 \times 3}$ is symmetric. If furthermore $\Theta(E(\mathcal{T})) \subset[0, \pi / 2]$, then the matrix is negative semidefinite of rank 2 . In particular, there is a concave function $W(u)$ defined on $\mathbb{R}^{V(\mathcal{T})}$ such that $\frac{\partial W}{\partial u_{i}}=a_{i}$. This implies that the discrete Ricci flow defined as

$$
\frac{d r(t)}{d t}(v)=-2\left(K_{r}(v)-\hat{K}(v)\right) r(t)(v)
$$

is the gradient flow of a concave function (namely $W$ ). From this fact, many of the basic properties, including long-time existence, of discrete Ricci flow follow. The flow is robust and alogrithmically effective if $\Theta(E(\mathcal{T})) \subset$ $[0, \pi / 2]$.

Discrete Ricci flow does not work well if one $\Theta(e)$ lies outside of the interval $[0, \pi / 2]$. This is one of the drawbacks of the flow for real-world applications. Many 


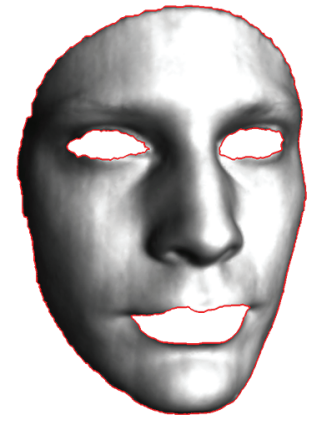

Figure 11. The extremal length of a topological quadrilateral.

polyhedral surfaces produced by digital media cannot be expressed as circle packing metrics such that $\Theta(E(\mathcal{T})) \subset$ $[0, \pi / 2]$. Modifications of the triangular meshes are needed to achieve this condition $\Theta(E(\mathcal{T})) \subset[0, \pi / 2]$.

The convergence of circle packing metrics on bounded simply connected domains to the Riemann mapping was first conjectured by Thurston in 1985 and proved in a celebrated paper by Rodin-Sullivan in 1987 [17]. However, convergence questions for nonplanar surfaces remain open.

Below are some examples of discrete Ricci flows. Figure 11 shows one example of computing the extremal length of a topological quadrilateral using discrete Ricci flow. Basically, we set the target curvature to be zero for all interior and boundary vertices, except the four corners, and set the target curvatures for the corners to be $\pi / 2$. Then we run Ricci flow to get the target metric, and isometrically embed the surface using the target metric to obtain the planar rectangle. Here the discrete curvature at a boundary vertex is defined to be $\pi$ less the sum of all angles at $v$.
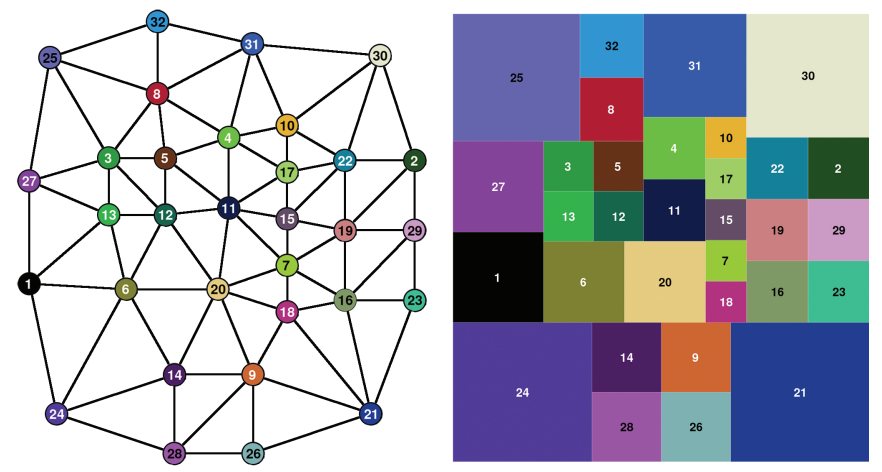

Figure 12. Square tiling of a 3-connected graph; each node is replaced by a square with the same label and color. Two nodes are connected in the graph if and only if their corresponding squares are tangent.

Figure 12 shows a generalization of circle packing by replacing circles by squares to compute the extremal length of a combinatorial quadrilateral. The left frame shows a 3connected graph, with four corner nodes. The right frame shows the extremal length, where each node is replaced
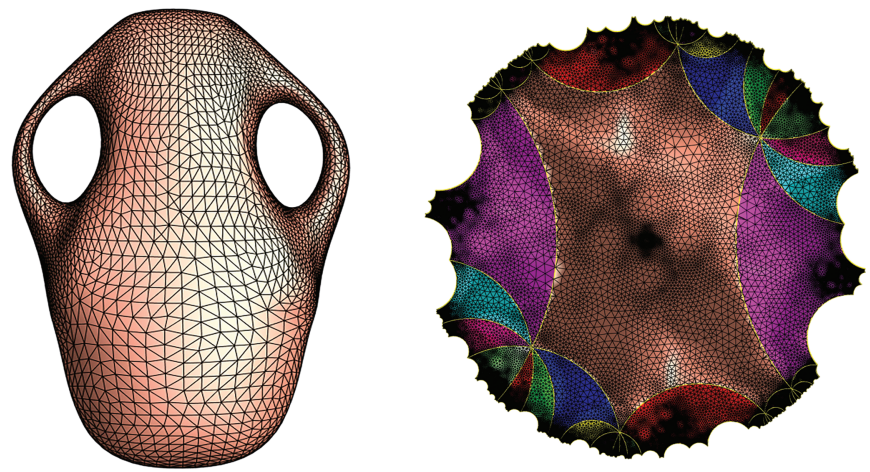

Figure 13. Uniformization for high genus surfaces.

by a square with the same label and color. Two nodes are connected in the graph if and only if their corresponding squares are tangent. In theory, squares can be replaced by more general convex shapes. Figure 13 shows an example for computing the hyperbolic metric on a high genus surface. As shown in the left frame, the input surface is triangulated, and each face is a hyperbolic triangle instead of a Euclidean triangle. The theoretic formulation and the algorithmic details are very similar. After obtaining the uniformization metric, we isometrically embed a finite portion of the universal covering space of the surface onto the Poincaré model of $\mathbb{R}^{2}$. Each color represents a fundamental polygon, and the boundaries of the fundamental polygons are hyperbolic geodesics.

Harmonic map. Another useful algorithm is based on surface harmonic maps for a genus zero closed surface [10]. Figure 14 shows the computational method for a genus zero closed surface: harmonic mapping. Intuitively, the harmonic energy measures the elastic deformation energy induced by a mapping between surfaces. It depends on the Riemannian metric of the target surface and the conformal structure of the source surface. Harmonic maps are the critical points of the harmonic energy within the homotopy class. If the target surface has negative Gaussian curvature,
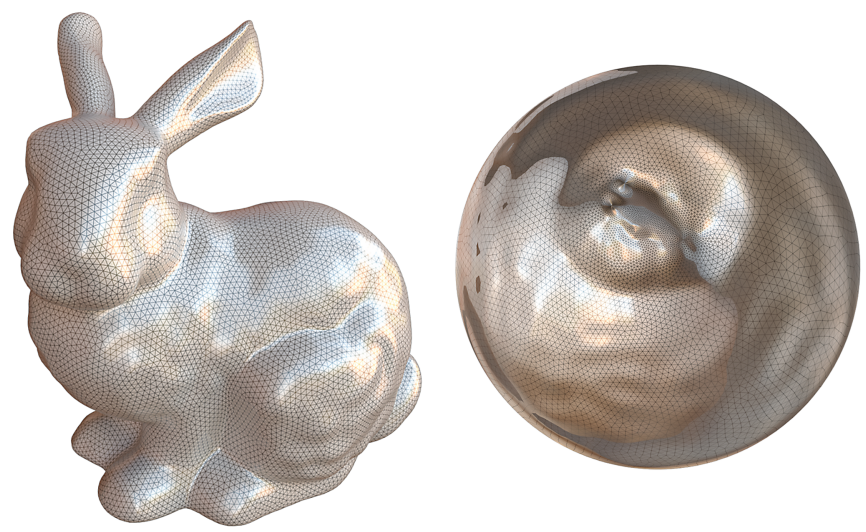

Figure 14. A spherical harmonic mapping from the Stanford bunny surface onto the unit sphere. 
and the mapping degree is one, then the harmonic mapping is diffeomorphic. A mapping between two surfaces induces the so-called Hopf differential on the source surface. If the mapping is harmonic, then its Hopf differential is a holomorphic quadratic differential on the source surface. Furthermore, if the Hopf differential is zero, then the mapping is conformal. Since holomorphic quadratic differentials on a genus zero surface must be zero, harmonic maps between genus zero closed surfaces must be conformal.

In practice, we first construct the Gauss map from the Stanford bunny surface to the unit sphere, then use the nonlinear heat diffusion method to reduce the harmonic energy to reach a harmonic. More specifically, we compute the Laplacian of the map and update the image of each point along the tangential direction of the Laplacian. Different harmonic maps differ by a Möbius transformation of the unit sphere, therefore we need to add an extra normalization constraint to ensure the uniqueness of the mapping. During the heat diffusion, we ensure that the mass center of the image of the surface on the unit sphere stays at the origin.
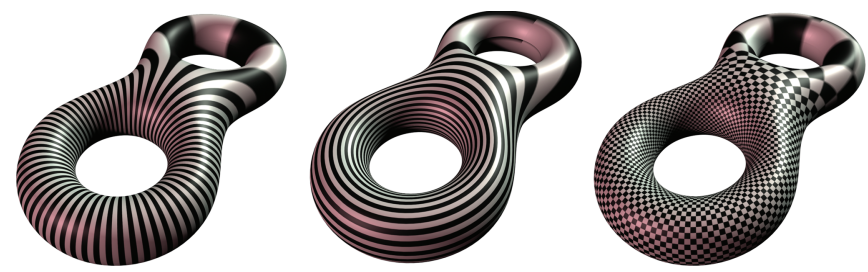

Figure 15. Two conjugate harmonic one-forms, in the left and middle frames, combine to form a holomorphic one-form in the right frame. The loops in the left (middle) frame show the vertical (horizontal) trajectories of the holomorphic form.

Hodge decomposition. Another algorithm is based on the Hodge decomposition theorem [11]. Hodge decomposition says that any differential form $\omega$ on a closed Riemannian manifold can be uniquely written as the sum of three parts: $\omega=d \alpha+\delta \beta+\gamma$, where $\gamma$ is harmonic, that is, $\Delta \gamma=0$ with $\Delta=d \delta+\delta d$. Intuitively, this can be interpreted as follows. Any vector field on a surface can be decomposed into three components: a curl-free part, divergence-free part and harmonic part. A vector field is harmonic if and only if it has zero curl and zero divergence, as shown in Figure 15.

In practice, we first compute the homology group basis of the surface $H_{1}(S, \mathbb{Z})$, then the cohomology group basis of $H^{1}(S, \mathbb{R})$. For each closed one-form $\omega$ representing a basis in $H^{1}(S, \mathbb{R})$, we find a function $f: S \rightarrow \mathbb{R}$, such that $\delta(\omega+d f)$ is zero. This is a linear elliptic geometric partial differential equation, and can be solved using the Finite Element Method generalized to the Riemannian surface setting. Then $\omega+d f$ is a harmonic one-form. Finally, the harmonic one-form paired with its conjugate form a holomorphic one-form. As shown in Figure 15, the left frame shows a harmonic one-form $\omega$, the middle frame shows the conjugate harmonic one-form $* \omega$, and the right frame shows a holomorphic one-form $\omega+\sqrt{-1} * \omega$. Using this method, we can construct the basis of the group of holomorphic one-forms of the Riemann surface. By linear combination, we can then construct any holomorphic one-form.
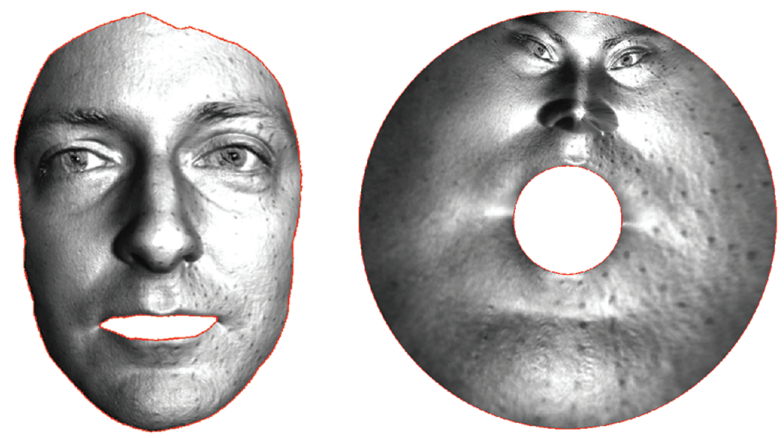

Figure 16. Topological annulus.

Suppose $S$ is a topological annulus with a Riemannian metric $\mathbf{g}$ and the boundary of $S$ are two loops $\partial S=\gamma_{1}-$ $\gamma_{2}$. We compute a holomorphic one-form $\omega$, such that the imaginary component of the integration of $\omega$ along $\gamma_{1}$ is $2 \pi$. Upon fixing a point $q$, the conformal mapping $\varphi(p)=$ $\exp \left(\int_{q}^{p} \omega\right)$ maps the surface onto a canonical annulus, as shown in Figure 16.
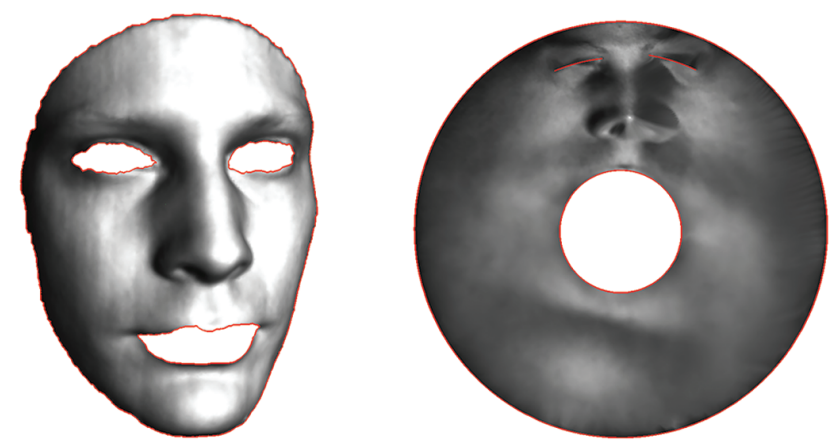

Figure 17. Circular slit mapping.

Suppose $S$ is a surface of genus zero with multiple boundaries, $\partial S=\gamma_{0}-\gamma_{1}-\gamma_{2}-\cdots-\gamma_{n}$; then $S$ is called a multiply connected annulus. We can construct a unique holomorphic one-form $\omega$, such that the imaginary part of the integration of $\omega$ along $\gamma_{0}$ is $2 \pi,-2 \pi$ along $\gamma_{1}$, and 0 along other boundary components. Then, upon fixing a base point $q$, the conformal mapping $\varphi(p)=\exp \left(\int_{q}^{p} \omega\right)$ maps the surface onto a canonical annulus with concentric circular slits, as shown in Figure 17. The algorithm was introduced in [21]. 

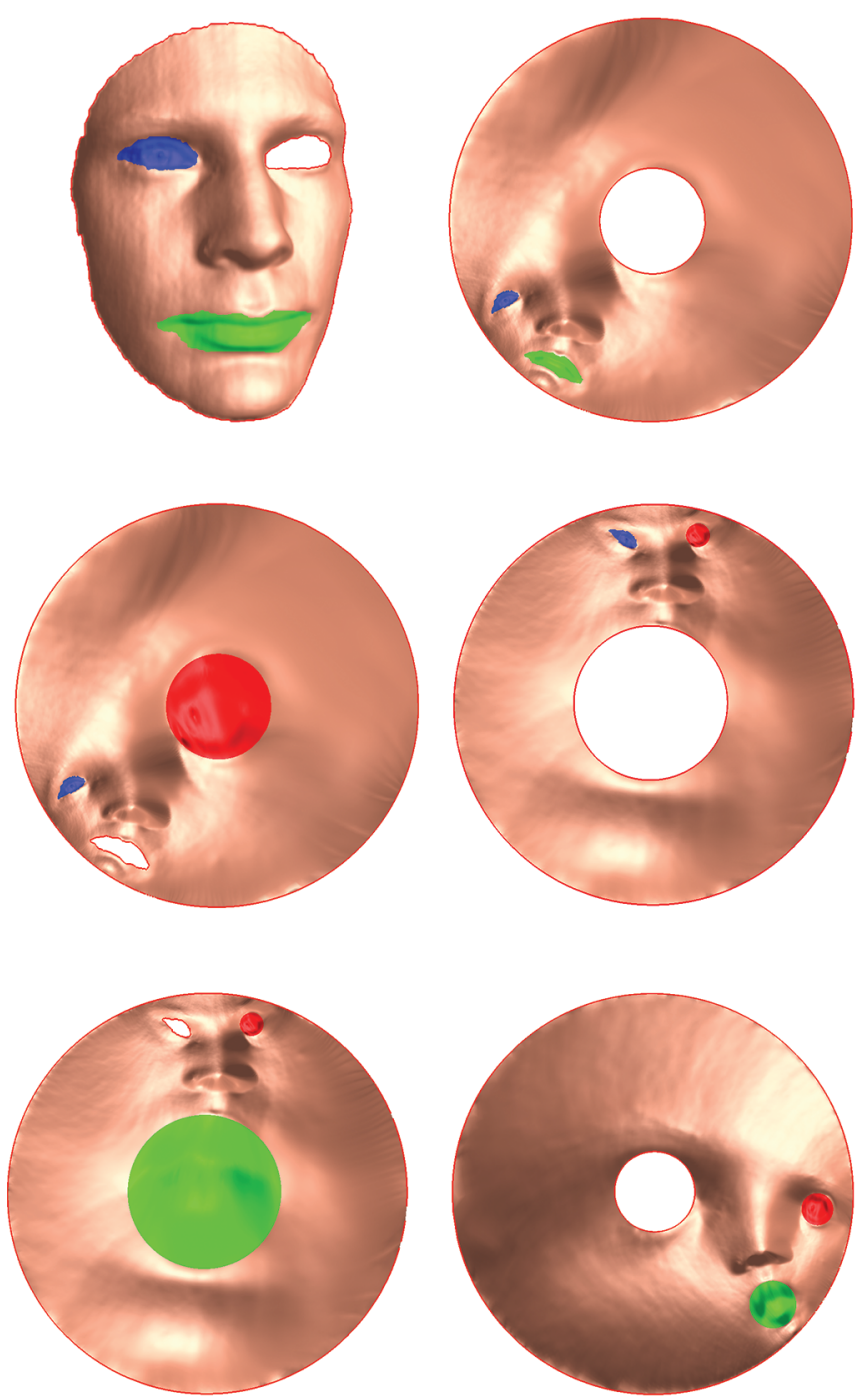

Figure 18. Koebe's iteration algorithm.

Figure 18 demonstrates Koebe's iteration algorithm [23] that conformally maps a multiply connected annulus onto a circle domain, namely the complement of the union of a finite number of disks. On the top row, we fill all the inner holes of the surface except one, then conformally map the topological annulus onto a canonical annulus; in the middle row, we fill the center circular hole and open another hole, then map the topological annulus onto a canonical annulus; at the bottom row, we fill the center circular hole and open the 3rd hole, and map the topological annulus onto a canonical annulus. We repeat this procedure, opening different holes in order and filling other holes, then map the topological annulus to the canonical annulus. The boundaries become rounder and rounder, and the image converges to a circle domain exponentially fast.
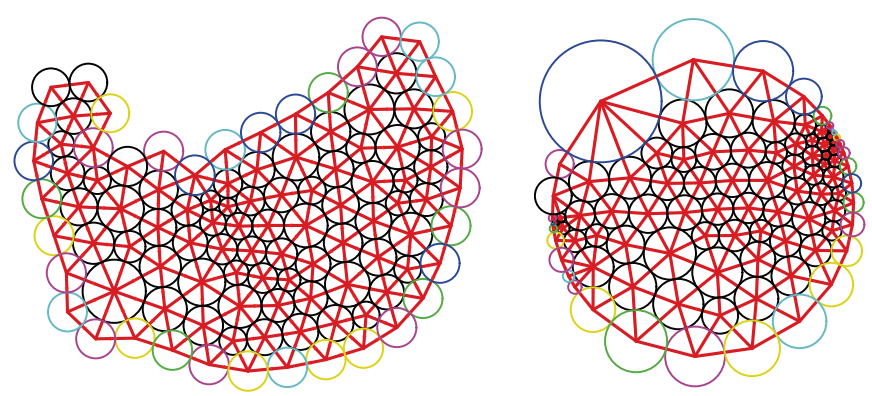

Figure 19. Discrete Riemann mapping using circle packing.

\section{Applications}

Computational conformal geometry has been broadly applied in many engineering fields. In the following, we briefly introduce some of our recent projects, which are the most direct applications of computational conformal geometry in the computer science field.

3.1. Graphics. Conformal geometric methods have broad applications in computer graphics. In computergenerated movies and games, shapes are modeled as triangular polyhedral surfaces, and the skins and clothes are represented as planar images, which are called texture images. Surface parameterization technique refers to the process of mapping a surface onto a planar domain. Once the surfaces are parameterized onto planar domains, the texture images are pulled back onto the surface to achieve a photorealistic visual effect, and this technique is called texture mapping. Texture mapping is one of the most fundamental techniques in the computer graphics field. Isothermal coordinates are natural for global surface parameterization purposes. Because conformal mapping doesn't distort the local shapes, it is desirable for texture mapping. Figure 20 shows an example of texture mapping constructed using holomorphic differentials.

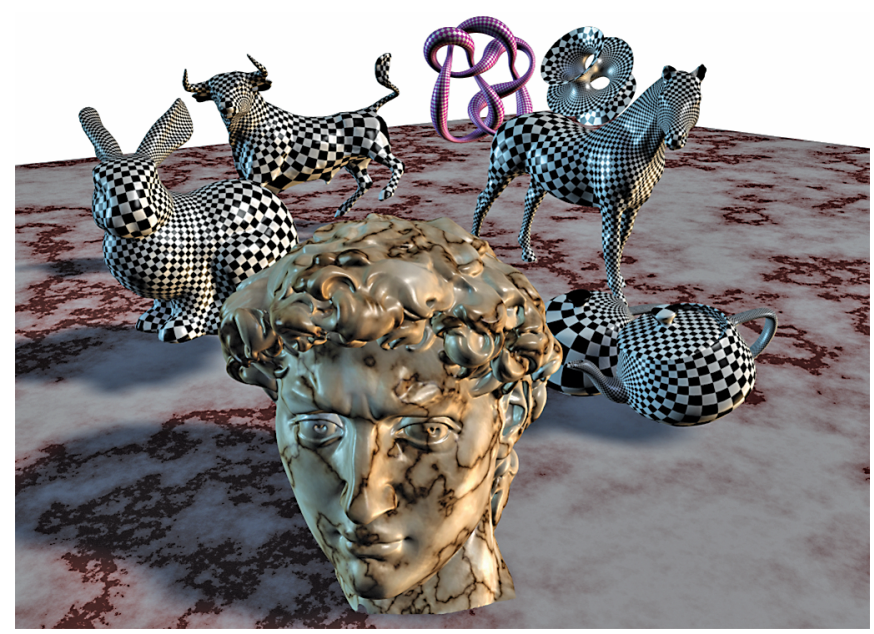

Figure 20. Global conformal surface parameterization for texture mapping. 

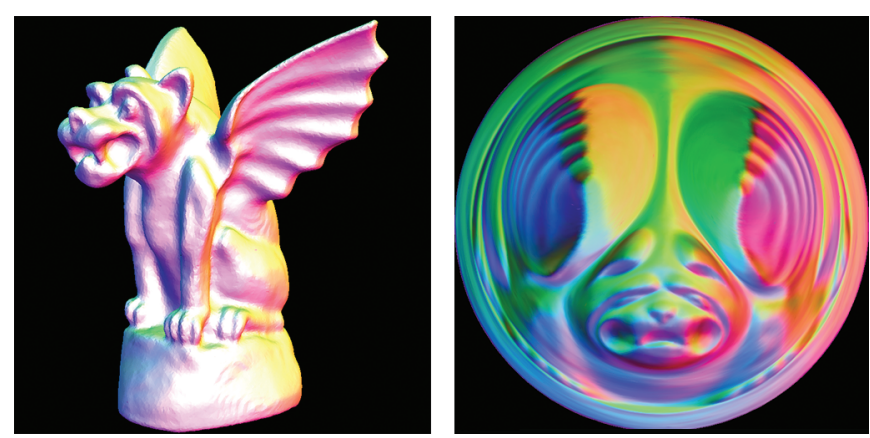

Figure 21. Normal map based on Riemann mapping and optimal transportation map.

In computer games, to improve the interaction speed, geometric representation of surfaces has to be simplified, namely, the number of faces is reduced. On the other hand, rough geometry jeopardizes the rendering quality. The commonly applied technique to solve this conflict is the normal map technique. This technique uses a texture image to represent the normal field of the surface. As shown in Figure 21, each pixel of the texture image in the right frame is mapped to a point on the surface, the red, blue and green values of the pixel represent the values of the $x-, y$ - and $z$-coordinates of the corresponding normal. The geometry of the gargoyle model is coarse, but its normal field is much refined. The rendering effect heavily depends on the resolution of the normal field. Hence the normal map technique offers a high-resolution normal field with a low-resolution geometry and achieves both good rendering quality and interaction speed. Again, the normal map of the gargoyle model is achieved by a Riemann mapping composed with an optimal transportation map, such that the parameterization is area preserving.
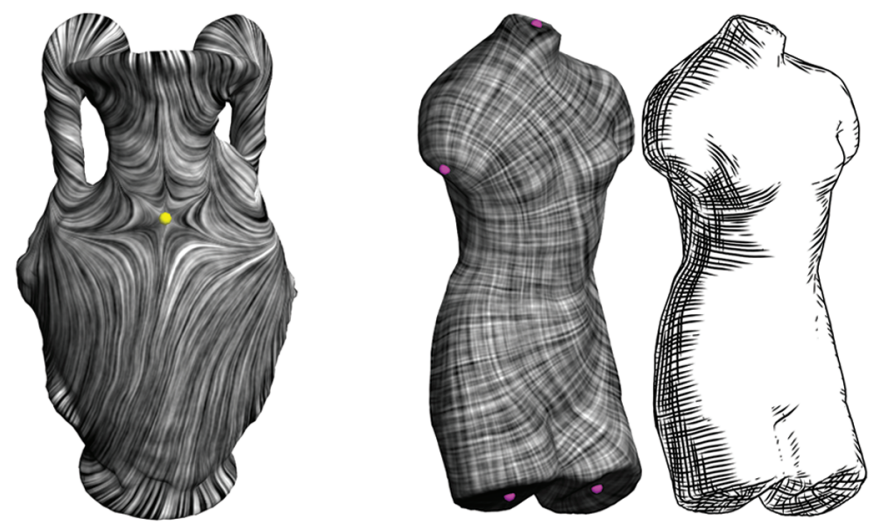

Figure 22. Vector field design using flat metrics.

For nonphotorealistic rendering and special art form design, people want to use computers to mimic artists to generate a pencil sketch or an oil painting. Generating an artificial sketch is equivalent to designing smooth vector fields. In practice, a user predefines the singularities, and then we can compute a flat metric with the prescribed singularities using Ricci flow. The smooth vector field can be generated by parallel transport of a tangent vector at the starting point, and using harmonic one-forms to compensate the holonomy of the metric. Figure 22 shows examples for vector field design on surfaces using the curvature flow method [22].


Figure 23. Facial surface matching by a Teichmüller map; all ellipses have the same eccentricity.

3.2. Vision. Surface matching is a fundamental problem in computer vision, which aims at finding a diffeomorphism between two surfaces with landmark constraints and least distortion. Figure 23 illustrates one example of matching two human facial surfaces. The surfaces are conformally mapped onto the planar unit disks by Riemann mappings. The major feature points are located based on the geometry and the texture information using either a geometric method or statistical learning method, which are shown as red dots. The problem of finding a diffeomorphism between facial surfaces is converted to finding one between the planar disks, and the latter one is much simpler and more efficient to compute.

Suppose $f: \mathbb{D}^{2} \rightarrow \mathbb{D}^{2}$ is an automorphism of the disk on the complex plane; then it satisfies the Beltrami equation

$$
\frac{\partial f}{\partial \bar{z}}=\mu \frac{\partial f}{\partial z}
$$


where $\mu$ is called the Beltrami coefficient of the mapping $f$. Geometrically, the mapping maps infinitesimal circles to infinitesimal ellipses. At each point $z \in \mathbb{D}^{2}$, the eccentricity of the ellipse is given by $\frac{1+|\mu|}{1-|\mu|}$, and the orientation of the ellipse is $\frac{1}{2} \arg \mu$. Therefore, the Beltrami coefficient describes the shape and the orientation of the ellipses, but not their size. If the eccentricity is bounded, then the mapping is called a quasi-conformal mapping. Amazingly, the generalized Riemann mapping theorem claims that we can recover the quasi-conformal mapping from its Beltrami coefficient uniquely up to a Möbius transformation by solving the Beltrami equation. Intuitively, if we change the Riemannian metric on the source from $|d z|^{2}$ to $|d z+\mu d \bar{z}|^{2}$, then the quasi-conformal mapping $f$ becomes conformal, and we can use conformal mapping algorithms to compute it. This algorithm is called the auxiliary metric method.

Assume $\mu_{1}$ and $\mu_{2}$ are two Beltrami coefficients, and $f^{\mu_{1}}$ and $f^{\mu_{2}}$ are their corresponding automorphisms of the disk. If $f^{\mu_{1}}$ and $f^{\mu_{2}}$ map the corresponding feature points to the same positions, then we say the two mappings are Teichmüller equivalent. The Teichmüller equivalence classes form the Teichmüller space; the geodesic connecting two classes in this space is given by the Teichmüller map between them. The distortion of a mapping $f$ is measured by the maximal eccentricity of the infinitesimal ellipses, which is equivalent to the $L^{\infty}$ norm of its Beltrami coefficient $\left\|\mu_{f}\right\|_{\infty}$. Within each Teichmüller equivalence class, the mapping $f$ that minimizes $\left\|\mu_{f}\right\|_{\infty}$ is called the extremal quasi-conformal map. Under mild conditions, the extremal quasi-conformal map is a Teichmüller map, whose Beltrami coefficient has a special form, $\mu=k \frac{\bar{\varphi}}{|\varphi|}$, where $\varphi$ is a holomorphic quadratic differential of the surface with feature points removed and $0 \leq k<1$ is a constant. All the infinitesimal ellipses induced by a Teichmüller map have the same eccentricity. The Teichmüller map can be achieved by optimization in the space of Beltrami coefficients [14]. Figure 23 shows a Teichmüller map between the two human facial surfaces. The mapping maps each feature point on the male face to the corresponding feature point on the female face. The Teichmüller map gives a diffeomorphism with the least angle distortion, and it can be applied for 3D facial recognition for surveillance purposes and for melanoma detection in dermatology.

Given a sequence of facial surfaces with dynamic expression change, the Teichmüller map can be used to find natural diffeomorphisms among them. The trajectories of the feature points give the facial expression, which can be transferred to other models for animation purposes. Figure 24 demonstrates an expression tracking result; the blue quadrilateral mesh is attached to the facial surface and moves along with it. The trajectories of the vertices of the blue mesh represent the expression [22]. The facial
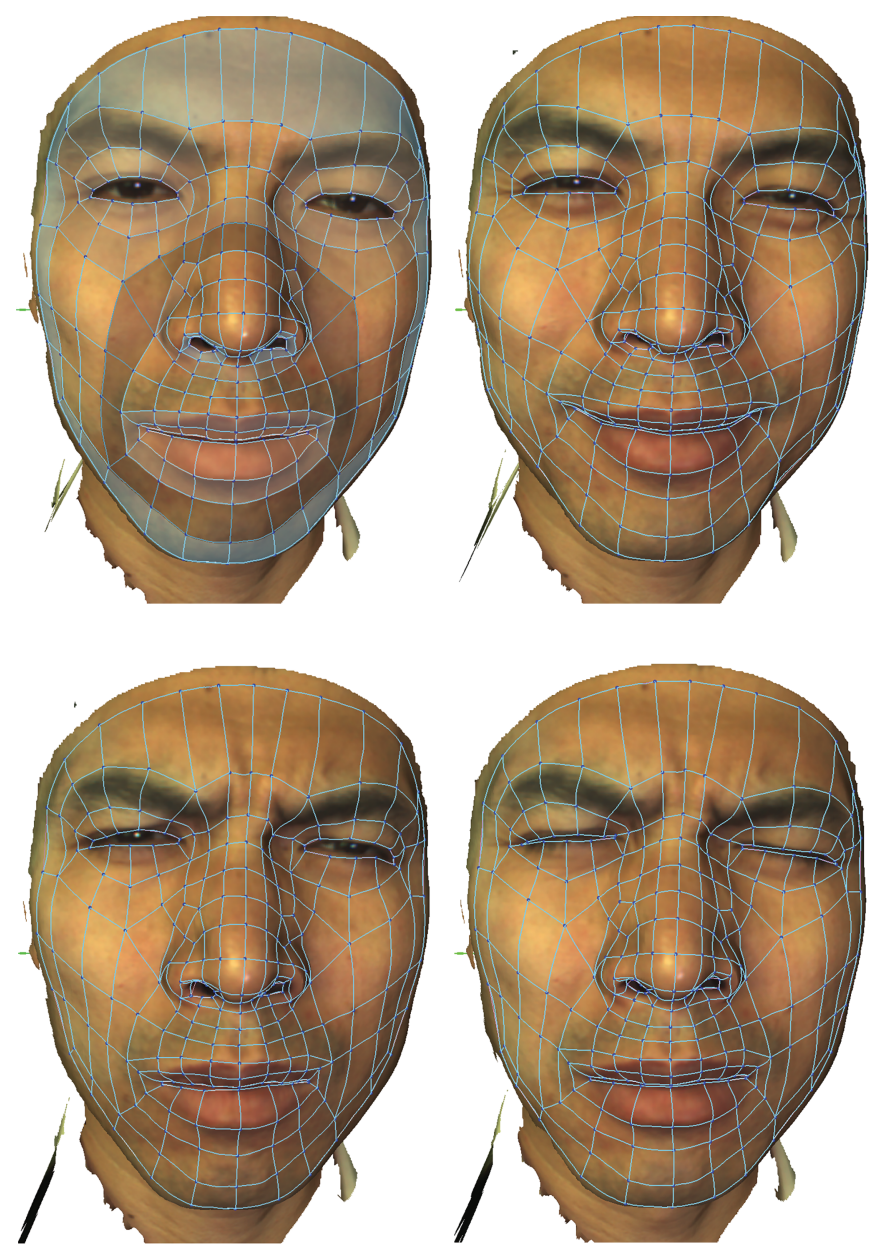

Figure 24. Facial expression tracking.

expression tracking technique plays an important role in the movie industry.

3.3. Wireless sensor network. Conformal geometry can also be used for networking applications. Figure 25 shows geometric routing for a wireless sensor network. The left frame shows a wireless sensor network, and each green dot represents a sensor, which can communicate with other sensors within a small neighborhood. Suppose every sensor has only local information, such as GPS coordinates of all the neighbors; there is no central sensor with global information. All sensors follow the same protocol to deliver messages distributively. One commonly used protocol is greedy routing: the sensor sends the packet to its direct neighbor, which is the closest one to the destination. If the network has concave holes, as shown in the left frame in Figure 25, the routing may get stuck at the nodes along the inner boundaries.

We can design a protocol to make the whole network compute a conformal map in a collaborative and distributive manner, such that the network is mapped onto a circle domain, as shown in the middle frame of the figure. Then the image of each sensor is used as the virtual coordinates, 

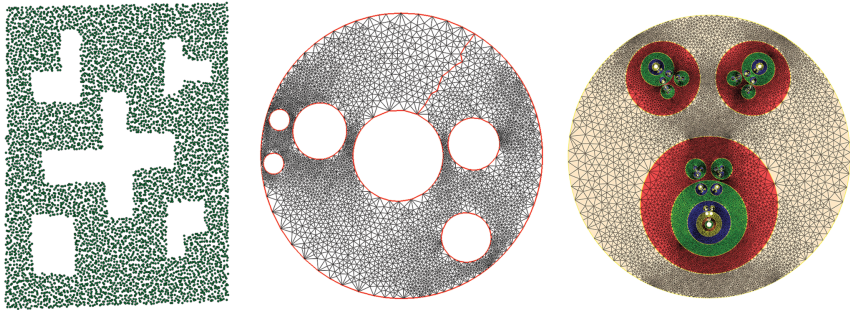

Figure 25. Conformal geometry for greedy routing and load balancing in a wireless sensor network.

on which the greedy routing is performed. Since all the boundaries become circles, greedy routing will guarantee the packet delivery [12].

Under greedy routing, all the routing paths are the shortest paths connecting the source and the target. Many shortest paths overlap with the inner boundaries. Therefore, the sensors on the inner boundaries will be overloaded, and their batteries will be exhausted much faster than others. In this scenario, we can reflect the whole network with respect to its inner circular boundaries, as shown in the right frame. The red layer is the image of the first reflection. The green, blue and yellow layers correspond to the second, third and fourth reflections. We can show that the number of inner circles increases exponentially fast with respect to the number of reflections, but the total area of the inner holes decreases exponentially fast. After several reflections, we obtain a covering space of the original network and perform the routing on the covering space using the virtual coordinates. This will balance the computational load among all the sensors.
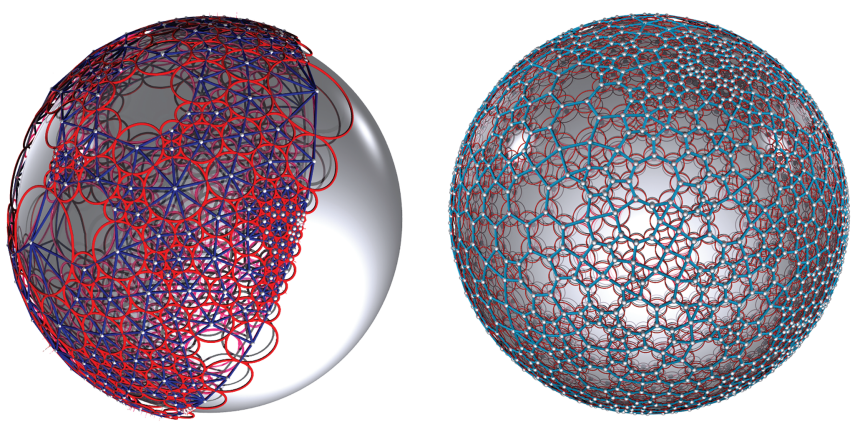

Figure 26. Graph embedding using circle packing.

For wireless sensor networks under water, since the GPS signal cannot penetrate the water, we can only get the connectivity information among the sensors, namely a graph representation of the network. For the purpose of routing, we need to embed the graph on some canonical spaces to obtain virtual coordinates for sensors. This graph embedding problem can be efficiently solved using conformal geometry as well. As shown in Figure 26, we compute the embedding of 3-connected planar graphs onto the unit sphere using the circle packing method. Basically, each node and
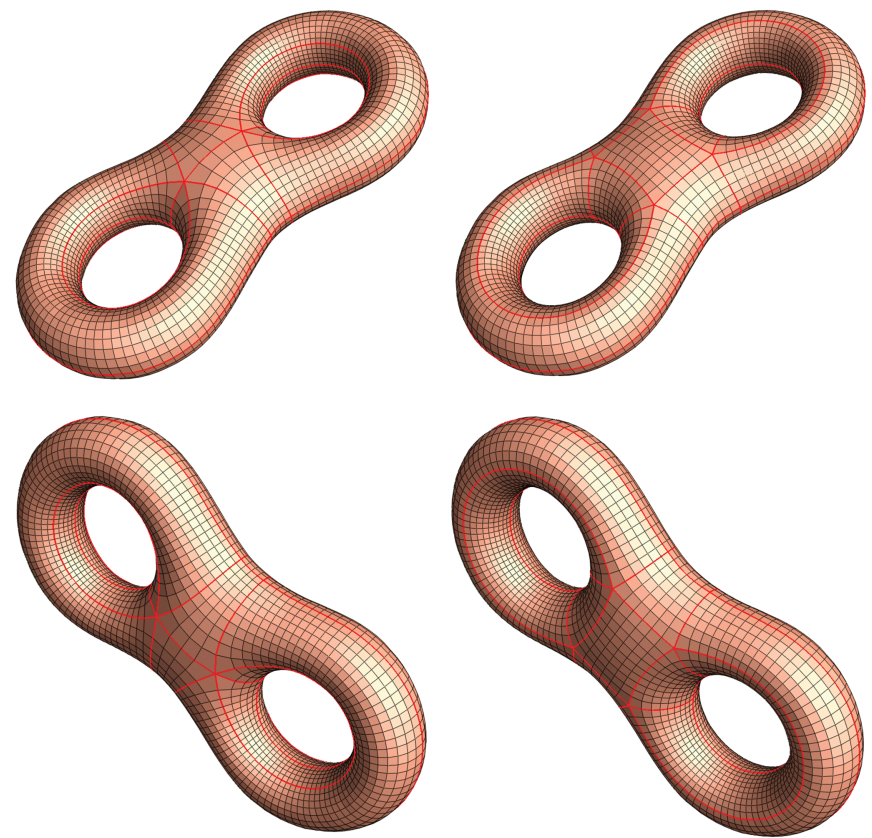

Figure 27. Quadrilateral mesh generation for a high genus surface.

face is associated with a circle; for each edge, the two circles associated with its end nodes are tangent to each other, the two circles associated with the faces sharing the edge are tangent to each other. Furthermore, for each face, the circle associated with the face is orthogonal to all the circles associated with its vertices. All such types of circle packings differ by a Möbius transformation of the unit sphere. Conventional methods, such as Tutte graph embedding, cannot handle nonplanar graphs. The conformal geometric method can embed them on the hyperbolic plane using circle packing similarly to that of the Euclidean case. This can be treated as a combinatorial version of uniformization. The sensor network can perform the routing based on this type of graph embedding [12].

3.4. Geometric modeling. In the conventional manufacturing industry, the representation of shapes needs to have higher-order continuity. The embedding of triangular meshes is only continuous but not differentiable. Therefore, the meshes need to be converted to piecewise polynomial representations with $C^{2}$ continuity almost everywhere, such as NURBS or T-Splines. The NURBS representation has local tensor product structure. In order to construct NURBS, we need to compute a quadrilateral tessellation of the input surface with high quality. This is called quadrilateral mesh generation.

Figure 28 shows different quadrilateral tessellations of a multiply connected planar domain. The vertices with topological valences other than 4 are called singularities. Figure 27 illustrates two quadrilateral meshes of a genus two surface. If we treat each face of a quad-mesh as the 



Figure 28. Different quadrilateral tessellations of a planar domain.

unit planar square, then the quad-mesh induces a flat metric with cone singularities. If this induced metric is conformal to the original surface metric, namely the two metrics differ by a scalar function at the normal (nonsingular) points, then the quad-mesh is conformal to the original shape, and it looks visually aesthetic. To construct conformal quad-meshes, the configuration of the singularities, including both the positions and indices (topological valences), is crucial. In fact, each conformal quad-mesh is associated with a meromorphic quartic differential of the input Riemann surface. The singularity configuration of the quad-mesh can be treated as the divisor of the meromorphic differential. The ratio between two such meromorphic quartic differentials is a globally defined meromorphic function on the original surface. The difference between the divisors of the differentials is the divisor of the function, which satisfies the so-called Abel condition. In practice, we can first find a divisor satisfying the Abel condition, then use Ricci flow to compute a flat metric with cone singularities. From the flat metric, we can construct the meromorphic differential. The horizontal and vertical trajectories of the differential generate the desired quad-mesh. The quad-meshes in Figure 28 and Figure 27 are produced this way. Once the conformal quad-mesh is obtained, we can build NURBS on it, as shown in Figure 29. In general, the continuity at the singularities will be reduced, and it is highly desirable to allow the users to prescribe the positions of singularities. This can be achieved using the above method based on Abel's condition and Ricci flow, as explained in [2].

3.5. Computer-aided manufacture. Conformal geometry also plays a fundamental role in the modern manufacturing industry. A foliation is a decomposition of a surface into a family of curves, such that locally it has tensor product structure. Figure 30 shows foliations on cat surfaces with boundaries and punctures. The cat surface is decomposed into a family of closed loops, and each loop is called a leaf of the foliation. Locally, the leaves are parallel to each other, and the bifurcations of the leaves are the singularities. Roughly speaking, two foliations are equivalent if a surface diffeomorphism homotopic to the identity

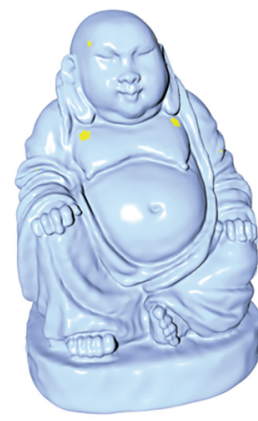

Spline surface

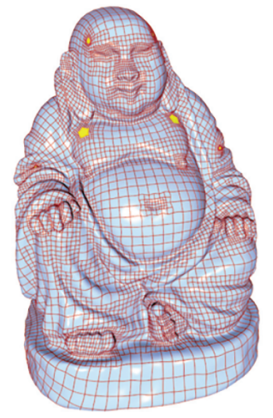

Knot structure

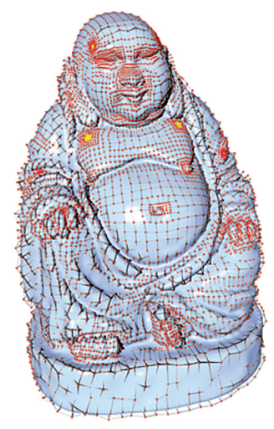

Control net
Figure 29. A spline surface with prescribed singularities (the centers of the yellow regions). The control net controls the shape, the knot structure describes the local chart of the atlas.

deforms one to the other. The horizontal trajectories of a holomorphic quadratic differential form a foliation. Inversely, for any foliation, there is a holomorphic quadratic differential whose horizontal trajectory foliation is equivalent to it. Based on this fact, we can construct all the foliations on a surface as in [13].

Figure 31 shows one way to compute a foliation on a high genus $g$ surface. First, we find $3 g-3$ disjoint simple loops. The loops divide the surface into $2 g-2$ segments, and each segment is a genus 0 surface with three boundary components, namely a pair of pants. This process is called a pants decomposition, as shown in the left frame on the top row. Then we construct a pants decomposition graph, such that each node corresponds to a pair of pants and each edge connecting two nodes represents a cutting loop shared by the corresponding two pairs of pants. Then we assign a length for each edge and compute a harmonic map from the surface to the pants decomposition graph. The preimage of each point on the graph is a leaf of the foliation, as shown in the right frame on the top row. The preimage of a node of the graph is a critical leaf of the foliation. All the critical leaves partition the surface into $3 g-3$ cylinders, as shown in the left frame in the bottom row. The Hopf differential of the harmonic map is a holomorphic quadratic differential whose horizontal and vertical trajectories give two conjugate foliations, as shown in the right frame in the bottom row. Using this method, we can compute conjugate foliations. Figure 32 shows the fabrication process of a human hand surface. Two conjugate foliations are computed. The leaves of one foliation are


Figure 30. Foliations on a cat surface with punctures. 

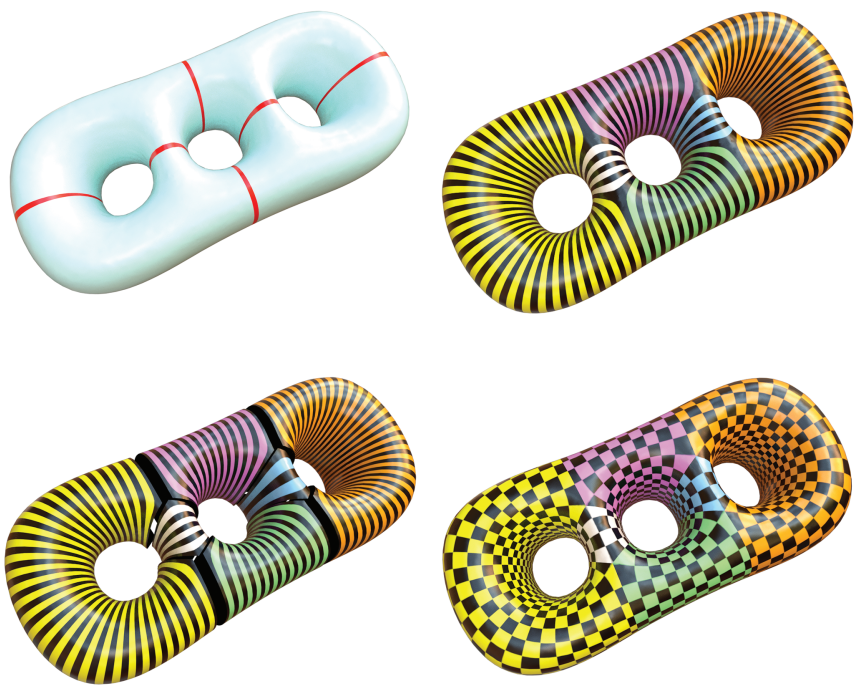

Figure 31. Computing holomorphic quadratic differentials and foliations via graph-valued harmonic maps.

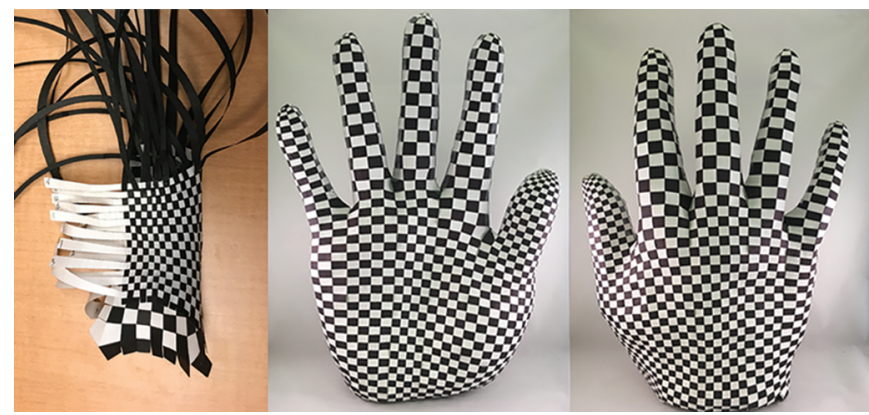

Figure 32. Fabricating shapes by weaving leaves of conjugate foliations.

white, and those of the other foliation are black. By weaving these two families of leaves, we can construct the hand shape. This gives a flexible means to fabricate surfaces with complicated geometries and topologies.

Figure 33 shows a meta-material design process on surfaces. Meta-materials don't exist in nature but are made of natural materials with specially designed micro geometric structures, such that they have desired mechanical, thermal or electro-magnetic properties. For example, a piece of rectangular rubber has a positive Poisson ratio. If we pinch it from the top and bottom sides, then the left and right sides will expand. If the left and right sides also shrink, then we say the material has a negative Poisson ratio. Most natural materials have positive Poisson ratio values, but we can design a meta-material with a negative Poisson ratio, as shown in the figure. We first tessellate the surface into quadrilaterals, then replace each quad-face by a blue cell shown in the circle; adjacent cells are with orthogonal orientations. The surface with a thin cell structure has a negative Poisson ratio, and when a bullet hits it, the mass will concentrate on the hitting point.

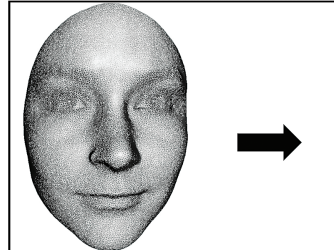

(a)



(d)

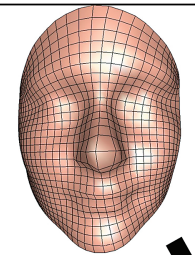

(b)



(c)
Figure 33. Meta-material design with negative Poisson ratio.
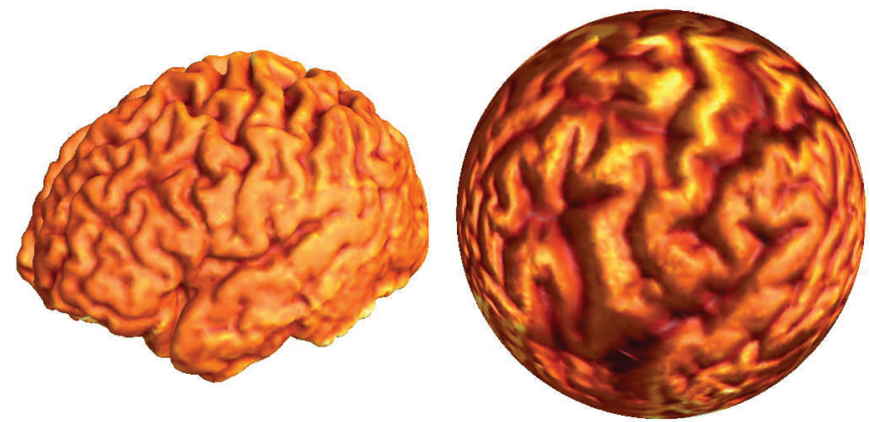

Figure 34. Brain spherical conformal mapping.

Therefore, by choosing the appropriate material, the facial mask can be printed out using 3D printing technology to get a bullet-proof mask.

3.6. Medical imaging. Conformal geometry has been applied to many fields in medical imaging. For example, in the field of brain imaging, it is crucial to register different brain cortex surfaces reconstructed from MRI or CT images. Cortex surfaces are topological spheres and can be uniformized onto the unit sphere conformally. Figure 34 illustrates this solution [12] by mapping brains to the unit sphere in a canonical way. Then, by finding an automorphism of the sphere, the registration between cortical surfaces can be easily established.

Another method is shown in Figure 35. The major landmark curves (succi and gyri) on the cortical surfaces are located, then the surface is sliced open along these landmarks and conformally mapped onto a circle domain. The centers and radii of the inner circles give the conformal module of the surface, which can be treated as the fingerprint of the cortical surface and used for classification and comparison. These methods have been applied for 

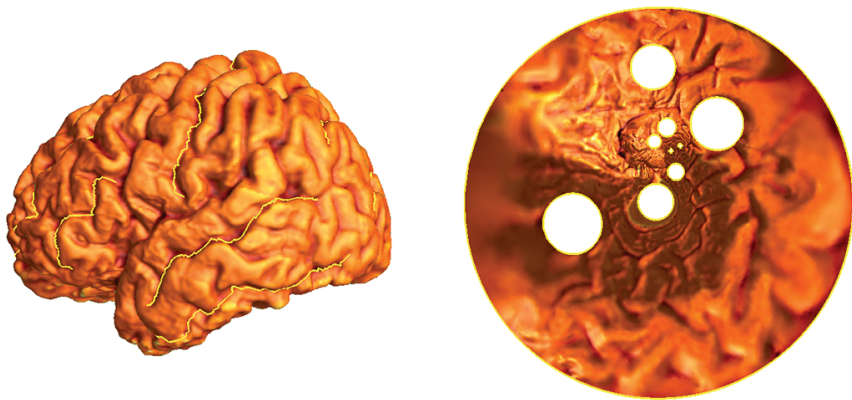

Figure 35. Brain morphology study using conformal module.
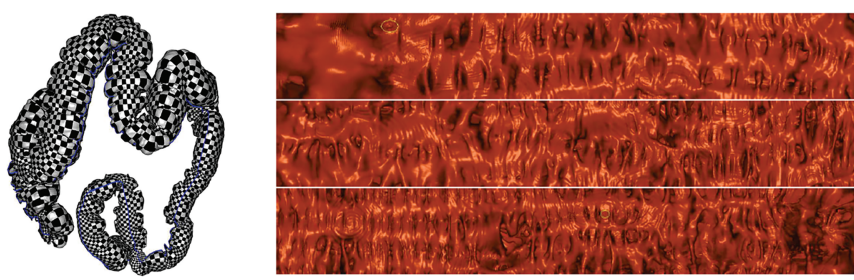

Figure 36. Colon conformal flattening.

neurological disease diagnosis, such as Alzheimer's disease, autism, Williams syndrome and so on.

Colon cancer is the third most common cause of cancerrelated death in the United States. The most effective way to prevent colon cancer is the colonoscopy. Conventional colonoscopy is invasive and may cause complications. Virtual colonoscopy is less invasive and with fewer complications. In virtual colonoscopy [22], the colon surface is reconstructed from CT images and analyzed using the geometric method. As shown in Figure 36 left frame, a colon surface has many haustral folds in anatomy, and when the polyps are hidden in these folds, they are hard to be located and recognized. By using conformal geometric methods, one can flatten the whole colon surface onto a planar rectangle, as shown in the right frame. Then all the haustral folds are expanded, all polyps are exposed, and abnormalities can be found efficiently on the planar image. Furthermore, in practice, the colon surface will be scanned twice with supine and prone positions. Because the colon surface is highly elastic, there will be large deformations between the two scans. Conformal colon flattening can be applied to find a good registration between the supine and prone colon surfaces [22]. Today, the conformal colon flattening technique has already been widely used in clinical practice.

\section{Conclusion}

Conformal geometry has many merits, which make it suitable for engineering and medical applications. Conformal mapping can reduce dimensionality and convert $3 \mathrm{D}$ geometric processing problems to $2 \mathrm{D}$ ones. The uniformization theorem unifies all kinds of shapes in reality and greatly simplifies the task of algorithm design.
Quasi-conformal mapping is capable of modeling all diffeomorphisms among compact surfaces and Teichmüller space models general shape space and give the metric to measure shape differences. The harmonic mapping matches surfaces with the least deformation. So far, surface discrete curvature flows are the only way to construct Riemannian metrics with prescribed curvatures. All these methods have achieved great successes in real applications, and some of them are used in daily life.

Despite the successes, there are still many fundamental open problems in computational conformal geometry, which will require deeper insights and more sophisticated and accurate computational methodologies. So far, most computational algorithms are based on the geometric analysis method and few of them are from algebraic geometry. We expect to see greater theoretic breakthroughs and broader applications in computational conformal geometry in the near future.

\section{References}

[1] Alexander I. Bobenko, Ulrich Pinkall, and Boris A. Springborn, Discrete conformal maps and ideal hyperbolic polyhedra, Geom. Topol. 19 (2015), no. 4, 2155-2215, DOI 10.2140/gt.2015.19.2155 MR3375525

[2] Wei Chen, Xiaopeng Zheng, Jingyao Ke, Na Lei, Zhongxuan Luo, and Xianfeng Gu, Quadrilateral mesh generation I: Metric based method, Comput. Methods Appl. Mech. Engrg. 356 (2019), 652-668, DOI 10.1016/i.cma.2019.07.023. MR3989935

[3] Bennett Chow and Feng Luo, Combinatorial Ricci flows on surfaces, J. Differential Geom. 63 (2003), no. 1, 97-129. MR2015261

[4] Yves Colin de Verdière, Un principe variationnel pour les empilements de cercles (French), Invent. Math. 104 (1991), no. 3, 655-669, DOI 10.1007/BF01245096 MR1106755

[5] François Fillastre, Polyhedral hyperbolic metrics on surfaces, Geom. Dedicata 134 (2008), 177-196, DOI 10.1007/s10711-008-9252-2 MR2399657

[6] David Glickenstein, Discrete conformal variations and scalar curvature on piecewise flat two- and three-dimensional manifolds, J. Differential Geom. 87 (2011), no. 2, 201-237. MR2788656

[7] Xianfeng Gu, Ren Guo, Feng Luo, Jian Sun, and Tianqi $\mathrm{Wu}$, A discrete uniformization theorem for polyhedral surfaces II, J. Differential Geom. 109 (2018), no. 3, 431-466, DOI $10.4310 / \mathrm{jdg} / 1531188190$ MR3825607

[8] Xianfeng David Gu, Feng Luo, Jian Sun, and Tianqi $\mathrm{Wu}$, A discrete uniformization theorem for polyhedral surfaces, J. Differential Geom. 109 (2018), no. 2, 223-256, DOI 10.4310/jdg/1527040872. MR3807319

[9] David Gu, Feng Luo, and Tianqi Wu, Convergence of discrete conformal geometry and computation of uniformization maps, Asian J. Math. 23 (2019), no. 1, 21-34, DOI 10.4310/AJM.2019.v23.n1.a2 MR3949589 
[10] Xianfeng Gu, Yalin Wang, Tony F Chan, Paul M Thompson, and Shing-Tung Yau, Genus zero surface conformal mapping and its application to brain surface mapping, IEEE Transaction on Medical Imaging (TMI) 23 (2004), no. 8, 949958.

[11] Xianfeng Gu and Shing-Tung Yau, Global conformal surface parameterization, Proceedings of the 2003 Eurographics/ACM SIGGRAPH symposium on geometry processing, Eurographics Association, 2003, pp. 127-137.

[12] Xianfeng David Gu and Shing-Tung Yau, Computational conformal geometry, Advanced Lectures in Mathematics (ALM), vol. 3, International Press, Somerville, MA; Higher Education Press, Beijing, 2008. With 1 CD-ROM (Windows, Macintosh and Linux). MR2439718

[13] Na Lei, Xiaopeng Zheng, Jian Jiang, Yu-Yao Lin, and David Xianfeng Gu, Quadrilateral and hexahedral mesh generation based on surface foliation theory, Comput. Methods Appl. Mech. Engrg. 316 (2017), 758-781, DOI 10.1016/j.cma.2016.09.044 MR3610120

[14] Lok Ming Lui, Ka Chun Lam, Shing-Tung Yau, and Xianfeng Gu, Teichmüller mapping (T-map) and its applications to landmark matching registration, SIAM J. Imaging Sci. 7 (2014), no. 1, 391-426, DOI 10.1137/120900186 MR3170503

[15] Feng Luo, Combinatorial Yamabe flow on surfaces, Commun. Contemp. Math. 6 (2004), no. 5, 765-780, DOI 10.1142/S0219199704001501, MR2100762

[16] Junfei Dai, Xianfeng David Gu, and Feng Luo, Variational principles for discrete surfaces, Advanced Lectures in Mathematics (ALM), vol. 4, International Press, Somerville, MA; Higher Education Press, Beijing, 2008. [Author name on title page: Junei Dai]. MR2439807

[17] Burt Rodin and Dennis Sullivan, The convergence of circle packings to the Riemann mapping, J. Differential Geom. 26 (1987), no. 2, 349-360. MR906396

[18] M. Roček and Ruth M. Williams, Quantum Regge calculus, Phys. Lett. B 104 (1981), no. 1, 31-37, DOI 10.1016/03702693(81)90848-0 MR627806

[19] Kenneth Stephenson, Introduction to circle packing: The theory of discrete analytic functions, Cambridge University Press, Cambridge, 2005. MR2131318

[20] William P. Thurston, Three-dimensional geometry and topology. Vol. 1, Princeton Mathematical Series, vol. 35, Princeton University Press, Princeton, NJ, 1997. Edited by Silvio Levy. MR1435975

[21] Xiaotian Yin, Junfei Dai, Shing-Tung Yau, and Xianfeng Gu, Slit map: conformal parameterization for multiply connected surfaces, Advances in geometric modeling and processing, Lecture Notes in Comput. Sci., vol. 4975, Springer, Berlin, 2008, pp. 410-422, DOI 10.1007/978-3540-79246-8_31. MR2497857

[22] Wei Zeng and Xianfeng David Gu, Ricci flow for shape analysis and surface registration: Theories, algorithms and applications, SpringerBriefs in Mathematics, Springer, New York, 2013. MR3136003

[23] Wei Zeng, Xiaotian Yin, Min Zhang, Feng Luo, and Xianfeng Gu, Generalized koebe's method for conformal mapping multiply connected domains, 2009 SIAM/ACM joint conference on geometric and physical modeling, ACM, 2009, pp. 89-100.

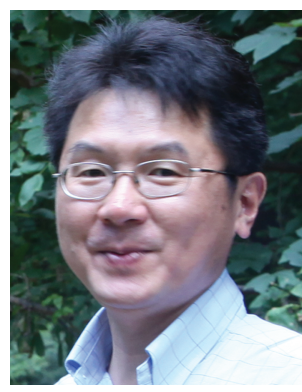

Xianfeng Gu



Shing-Tung Yau

Credits

All images are courtesy of the authors.

Photo of Xianfeng Gu is courtesy of Li Yan.

Photo of Feng Luo is courtesy of Feng Luo.

Photo of Shing-Tung Yau is courtesy of Michael Eichmair. 